Bundesgesundheitsbl - Gesundheitsforsch Gesundheitsschutz 2009 52:239-255 DOI 10.1007/s00103-009-0783-y Online publiziert: 12. Februar 2009 (c) Springer Medizin Verlag 2009

\section{Empfehlungen für den Umgang mit Beobachtungen von räumlich-zeitlichen Krankheitsclustern}

\author{
Mitteilung der Kommission „Methoden und \\ Qualitätssicherung in der Umweltmedizin“"
}

\section{Fragestellungen und Ziele}

Die Frage nach den Ursachen für aufgetretene Erkrankungen wird im Arzt-Patienten-Gespräch häufig gestellt. Der Dialog über mögliche Krankheitsursachen macht die Erkrankungen besser verständlich und ermöglicht gegebenenfalls eine Prävention. Wird eine zeitliche oder räumliche Häufung von ernsten Krankheitsfällen in der Bevölkerung wahrgenommen, so ist jede öffentliche Frage nach einer möglichen gemeinsamen Ursache eine ebenso berechtigte wie verantwortungsbewusste Reaktion. Eine Ausweitung oder eine zeitliche Verlängerung des Auftretens von adversen Gesundheitseffekten [1] in der Bevölkerung zu vermeiden, ist Teil des öffentlichen Auftrages. Den rechtlichen Rahmen geben hierbei die Landesgesundheitsgesetze. Dies ist beispielsweise formuliert als „Der öffentliche Gesundheitsdienst ergreift bei begründetem Verdacht einer Gefährdung geeignete Maßnahmen zur Aufklärung von möglichen gesundheitlichen Auswirkungen. Er kann hierzu Untersuchungen und Messungen selbst durchführen oder durch Auftrag vergeben, die auf die Ermittlung der Exposition, der Belastung des menschlichen Organismus sowie gesundheitlicher Risiken und Beeinträchtigungen abzielen“ [2]. Das Beseitigen, Beschränken bzw. Mindern von bestehenden, bekannten oder begründet als ursächlich angenommenen Einfluss- und Risikofaktoren ist Teil des erforderlichen Risikomanagements.

Mit dieser Handreichung möchte die Kommission „Methoden und Qualitätssicherung in der Umweltmedizin“ des Robert Koch-Instituts (RKI) zum einen Ärzte/Ärztinnen und umweltmedizinisch Tätige oder Interessierte ansprechen und ermutigen, bei festgestellten oder nach den vorliegenden Daten vermuteten räumlich-zeitlichen Erkrankungshäufungen (Cluster) frühzeitig und sachgerecht zuständige Institutionen zu informieren. Zum anderen sollen die Einrichtungen des öffentlichen Gesundheitsdienstes (ÖGD) informiert werden, wie sie in solchen Situationen systematisch und nach einer verlässlichen Methodik, gegebenenfalls unter Beteiligung weiterer kompetenter Stellen, vorgehen können. Auch wenn Clusterverdachtsmeldungen in den Bundesländern der Bundesrepublik Deutschland jeweils in unterschiedlichen Institutionen und Organisationsstrukturen bearbeitet werden müssen, wird eine vereinheitlichte gestufte Handlungsweise empfohlen, die an die jeweiligen Bedingungen angepasst werden kann. Für alle am Prozess der Klärung beteiligten Personen, Gruppen, Verbände und Institutionen möchte die RKI-Kommission einen Handlungs- und Ablaufrahmen vorschlagen, der eine sachgerechte Aufnahme/Dokumentation der erforderlichen Daten, Methoden und wesentlichen Bewertungskriterien beschreibt. Hierdurch soll ein präventives, zielgerichtetes und effizientes Handeln für den Umgang mit der Beobachtung von räumlich-zeitlichen Auffälligkeiten von unerwünschten Gesundheitseffekten unterstützt werden. Wenn es mit diesem Beitrag gelingt, die erforderlichen Informationsgrundlagen $\mathrm{zu}$ verbessern und die Kommunikation der Beteiligten untereinander zu erleichtern, wären einige der Kommissionsziele bereits erreicht.

\section{Begriffsdefinitionen}

Der unscharfe Begriff Cluster (engl.) wird in unterschiedlichen Fachgebieten unterschiedlich genutzt, er ist in diesem Kontext am besten mit den deutschen Begriffen: „Anhäufung, Häufung, Bündelung, Ballung “ zu umschreiben.

In dieser Arbeit soll unter "Meldung“, „Clustermeldung“"bzw. „Clusterverdachtsmeldung" der Hinweis auf eine gesehene oder vermutete zeitliche, räumliche oder gruppenspezifische Häufung von Erkrankungen verstanden werden.

„Risikokommunikation“ bezieht sich auf den gesamten Prozess von der Erstmeldung/Erstinformation über einen Clusterverdacht bis zum Abschlussgespräch. Risikokommunikation [3] soll dabei als interaktiver Prozess eines Informationsaustausches zwischen Personen, Gruppen und Institutionen verstanden 
werden, der sich (a) auf Aussagen und Nachrichten über die Natur von Risiken, (b) auf Ängste, Befürchtungen und Meinungen sowie (c) auf die institutionelle Bewertung und den Umgang mit Risiken bezieht. Die Kommunikation über bestehende Risiken steht hierbei im Vordergrund, sie darf nicht im Sinne von „Risiken ausreden" missverstanden werden.

\section{Anlässe und Ausgangssituationen}

Ein Anlass für eine Clusteruntersuchung ist zumeist die Beobachtung einer vermuteten Häufung von z. B. Erkrankungen, Fehlbildungen oder Todesfällen (Clustervermutung). Eine solche Untersuchung [4] stellt insofern eine Reaktion auf eine Meldung bzw. auf eine besorgniserregende Situation dar. Ausgangspunkt kann auch eine bestimmte Exposition sein, für die ein erhöhtes Erkrankungsrisiko vermutet wird.

Im Falle eines gehäuften Auftretens von Infektionskrankheiten (Endemie, Epidemie, Pandemie) ist die Vorgehensweise durch das Infektionsschutzgesetz und bestehende seuchenhygienische Maßnahmenpläne geregelt. Der Umgang mit Clustern infektiöser Genese ist deshalb nicht Gegenstand dieser Empfehlung. Die Methodik des Vorgehens hat jedoch eine Reihe von vergleichbaren Elementen und eine ähnliche Ablaufstruktur. Die zu stellenden Anforderungen an den Umgang mit vermuteten oder identifizierten räumlich-zeitlichen "Clustern" sind in jedem Fall hoch: Die Information muss von den angesprochenen Institutionen fachlich kompetent, schnell und von der Öffentlichkeit nachvollziehbar gegeben werden. Dies erfordert neben einem hohen fachlichen Standard immer auch eine gute Risikokommunikation.

Den Gesundheitsbehörden wird empfohlen, von Beginn an in einer kleinen Gruppe multidisziplinär zu arbeiten und insbesondere epidemiologischen und umweltmedizinischen Sachverstand hinzuzuziehen. Bewährt hat sich dabei, vorbereitend in einem Aktionsplan Ablauforganisation, Meldewege und Datenmanagement festzulegen und eine Arbeitsgruppe („Task-Force“) zu benennen, die bei Clusterhinweisen zeitnah aktiviert werden kann.
Der Klärungsprozess zur Beurteilung einer zeitlich-räumlichen Häufung von unerwünschten Ereignissen umfasst die Schritte des Dokumentierens, d. h. die Aufnahme der Datenlage, die Betrachtung und die fachliche Einordnung von möglichen Risikofaktoren, die Schritte des Analysierens (methodische Bearbeitung der Daten) und des Bewertens der Ergebnisse (statistische und fachliche Beurteilung der Situation). Die koordinierende Aufgabe liegt hierbei in der Zuständigkeit des ÖGD, der solche über den Routinebetrieb deutlich hinausreichenden Aufgaben oftmals jedoch nur in Kooperation mit weiteren Institutionen, der niedergelassenen Ärzteschaft und anderen Beteiligten meistern kann.

Einige Beispiele für „entdeckte“ und öffentlich schnell kontrovers diskutierte Cluster aus den letzten Jahren lassen annehmen, dass nicht immer die notwendige Sachkenntnis vorhanden war, um die vorliegenden Daten angemessen einzuordnen und um vorschnelle oder falsche Schlussfolgerungen zu vermeiden. Viele in der Presse zu findende "Clustermeldungen" hielten einer fachlich-methodisch begründeten Bewertung nicht stand. Andererseits werden gehäufte Krankheitsfälle meist zunächst erst durch Beobachtungen und Vermutungen von Bürgern und Bürgerinnen oder von einzelnen Ärzten/Ärztinnen als auffällig wahrgenommen. Solche Beobachtungen und Hinweise sind als Alarmsignale im Sinne eines Frühwarnsystems sehr wichtig.

Zeitlich-regionale Hinweise beziehen sich derzeit erfahrungsgemäß häufiger auf seltene schwere Erkrankungen, auf bestimmte Todesursachen wie Krebs oder auf Fehl- und Missbildungen [5]. Die Erstbeschreibung einer solchen Häufung ist meist noch unscharf formuliert: Der Beobachtungszeitraum ist oft unklar, und die Gruppenbildung kann beispielsweise Bewohner einer Region (Gemeinde, Stadtteil, Wohnsiedlung) oder eines Gebäudes, Mitarbeiter eines Betriebes, Personen im Einwirkungsbereich eines Emittenten oder Versorgers oder eine gemeinsame Freizeitaktivität betreffen. Weiterhin ist oftmals weder die genaue Zahl der Erkrankungsfälle noch die Größe der Bezugsbevölkerung bekannt.
Eine Fallhäufung kann dabei als räumliche (spatiale) oder als zeitliche (temporale) Häufung auffallen. Kleinräumige Krankheitshäufungen wirken oft besonders beunruhigend. Sie führen bei Betroffenen und den Nachbarn nicht selten zu einer kausalen Zuordnung zu möglichen Umweltexpositionen oder zu lokalen Besonderheiten (z. B. atomare Anlage, Industrie, Mülldeponie, Radar-/Funkanlagen; Innenraumluftschadstoffe). Die Information über Clustervermutungen wird dann oft mit der Forderung nach einer Ursachenaufklärung verbunden. In einzelnen Fällen wird aber auch anlassbezogen gezielt nach räumlichen und zeitlichen Clustern gesucht, wenn die Besorgnis wegen einer neuen oder bestehenden Anlage groß ist; die Beobachtungen sind hier von einer Hypothese geleitet, dass eine technische Anlage in der Wohnumgebung krank machen könnte (z. B. Mobilfunksender).

Entsprechende Anfragen und Hinweise müssen von den zuständigen Stellen bezüglich ihrer räumlichen und zeitlichen Dimension einerseits und im Vergleich zu „normalerweise“ zu erwartenden Fallzahlen bei gegebenen Bevölkerungs- bzw. Betroffenengruppengrößen andererseits eingeordnet werden. Die Bestimmung der Erwartungswerte für „normalerweise“ auftretende Fallzahlen hängt neben der Alters- und Geschlechtsstruktur direkt von der Grundrate der Erkrankung und von der Populationsgröße ab. Die „Norm“ ergibt sich aus der Betrachtung der spezifischen Erkrankungen im größeren Maßstab (z. B. alters- und geschlechtsspezifische Inzidenz-, Mortalitäts-, Fehlbildungsraten der Bundesrepublik Deutschland oder der Bundesländer). Die „normalerweise“ zu erwartende Anzahl von Fällen berechnet man im Allgemeinen auf der Grundlage dieser Raten für eine Region und unter Bezug auf die entsprechende Bevölkerung in einer definierten Zeitspanne. Im Sinne einer „Nullhypothese“ wird dabei von einer räumlich und zeitlich homogenen Verteilung des Erkrankungsrisikos ausgegangen. Die Centers for Disease Control and Prevention (CDC, Atlanta/USA) definieren ein Cluster [6] als „eine Zahl von aufgetretenen Fällen, die höher liegt, als sie in einer Personen- 
gruppe in einem geographischen Areal über einen bestimmten Zeitraum zu erwarten war".

Eine gebietsbezogene Clusterbeschreibung, z. B. „Die Region X zeigt extreme, auffällige, ungewöhnlich hohe, besorgniserregende Erkrankungsraten", kann dabei unterschiedliche geographische Bezüge haben. Sie kann sich ebenso auf frei gewählte Gebiete (z. B. Straßenzüge, Wohnsiedlungen, Nachbarschaften zu möglichen Emittenten wie auch auf administrative Gebietseinheiten (Gemeinden, Landkreise oder Stadtbezirke) beziehen. Gesehene zeitliche Häufungen haben einen Raumbezug wie räumliche Häufungen auch immer einen Zeitbezug haben. Stetige zeitliche Entwicklungen der Inzidenz, die die betrachtete Gesamtregion betreffen, werden dabei nicht als Cluster auffallen. Bei solchen regionalen Clusterhinweisen können (a) die Höhe der Erkrankungsrate einer Region, (b) die gemeinsam hohen Raten in mehreren benachbarten Regionen, (c) die räumliche Konzentration von Regionen mit hohen Erkrankungsraten im Umfeld vermuteter Verursacher/Risikoquellen oder (d) die zeitliche Häufung in einer Region im Vordergrund stehen.

Hiervon zu unterscheiden sind die systematischen Cluster-Untersuchungen, die auf der Grundlage von regionalisierten Daten aus bevölkerungsbasierten Erkrankungsregistern erfolgen. Hier werden z. B. die in den Gemeinden/Kreisen beobachteten Fallzahlen mit den Erwartungswerten verglichen (Heterogenität: Bewertung der Gleichheit des Erkrankungsrisikos über die Gebiete) und gegebenenfalls zusätzlich auf das Vorliegen von Tendenzen zu einer räumlichen Nachbarschaft von Gebieten mit höheren Erkrankungsraten (regionale Autokorrelation) geachtet. Den Auffälligkeiten wird hierbei durch Betrachtungen der Zeitreihen (zeitliche Entwicklung des Clusters), durch die Analyse auf Clusterzentren (Identifizierung von Risikogebieten) und ggf. durch umweltepidemiologische Untersuchungen zur Ursachenaufklärung nachgegangen.

Wird die Wohnadresse, ein Aufenthalts- oder Arbeitsort als Ausgangspunkt eines Verdachts auf regionale Häufungen gesehen, so wird in der Regel die "geringe regionale Distanz“ bzw. die „große räum- liche Nähe" der inzidenten oder prävalenten Fälle akzentuiert werden. Wird mit dem Clusterhinweis eine ursächliche Annahme bezüglich einer lokalisierbaren Risikoquelle verknüpft, so wird die regionale Verteilung der Punktdaten in eine räumliche Beziehung zur geografisch verorteten Punktquelle (z. B. Sendemasten, Industrieanlagen, Emissionsquellen), zu Linienquellen (Straßen, Schienen, Hochspannungstrassen) oder zu Flächenquellen (z. B. Gebiete erhöhter Boden-, Staub-, Luftbelastung) gesetzt.

Von einem „Cluster“ sollte, unabhängig von der Methodik der Beschreibung der Fälle, erst dann gesprochen werden, wenn in einer Region in einem definierten Zeitintervall eine Häufung von Fällen einer definierten Krankheit oder Todesursache oberhalb der Erwartungshäufigkeit vorliegt oder wenn mehrere benachbarte Gebiete/Regionen einen systematischen Trend zu höheren Erkrankungsraten aufweisen oder wenn die Fälle eine gegenüber der Erwartung sehr geringe räumliche Distanz und zeitliche Differenz zueinander aufweisen. Die Prüfung auf Vorliegen einer räumlich-zeitlichen Häufung (Cluster) impliziert ein sorgfältiges, methodisches Vorgehen in mehreren Stufen:

- Dokumentieren der Information aus dem ersten Kontakt,

- Aufnahme der Daten zum Clusterverdacht und

- parallel Bewertung der umweltbezogenen Gefahrenlage mit ggf. schneller Abwehr akuter Gefahren,

- Einordnung der Beobachtungen: Vorläufige Evaluation,

- Kommunikation der Ergebnisse (ob Clusterverdacht oder nicht)

- bei Clusterverdacht vertiefte Untersuchung.

Alle Schritte sollten durch angemessene Risikokommunikation begleitet werden.

Als Erstes sollte eine Verifizierung erfolgen, ob tatsächlich ein Cluster vorliegt. Dies setzt eine Falldefinition mit Ein- und Ausschlusskriterien mit einer räumlichen und zeitlichen Begrenzung voraus.

Clusterverdachtsklärungen sind daher nicht zwingend angezeigt, wenn bereits bei der Betrachtung der aufgelisteten Fälle erkennbar ist, dass eine niedrige Zahl von
Fällen verschiedenster Entitäten aus einem unscharf abgegrenzten Gebiet und/oder einem nicht präzisierten Zeitraum berichtet wird. Die Wahrnehmung einer ,allgemein erhöhten Krebsrate“ kann in vielen Fällen auch durch eine fachlich fundierte Kommunikation über die alters- und geschlechtsabhängige Häufigkeitserwartung von Krebserkrankungen (z. B. in Wohnregionen mit einem hohen Anteil älterer Bürger/innen) sachlich eingeordnet werden. Die Anfragen/Gespräche sollten gleichwohl bezüglich des Anlasses und der Beratungsergebnisse dokumentiert werden.

Zur Bewertung, ob es sich um eine Auffälligkeit handelt, sind immer Vergleichsdaten erforderlich. Sofern ein Vergleichskollektiv mit unmittelbarem räumlichem Bezug zur Clusterregion nicht herangezogen werden kann, muss ein Referenzkollektiv in die Untersuchungen einbezogen werden. Parallel dazu wird die Expositionsseite in einer umweltmedizinischen Bewertung im Hinblick auf mögliche Quellen und Noxen betrachtet. Plausible Umweltfaktoren, die von Betroffenen als mögliche Ursache vermutet werden, sollten auch ohne vorherige Bestätigung eines Clusters geprüft und bewertet werden.

Die geplanten und ergriffenen Schritte der Abklärung und die jeweils erreichten Ergebnisse sind den Betroffenen und Interessierten mitzuteilen (Risikokommunikation). Die meisten Fälle von vermuteten Clustern erfordern keine weiterführenden Untersuchungen, weil die beobachteten Fallzahlen im Rahmen der Erwartung liegen. Werden jedoch weiterführende Studien - beispielsweise bei einem nachgewiesenen Cluster - als notwendig angesehen, kann das Design einer geplanten Studie der vermuteten räumlichen und zeitlichen Expositionsentwicklung angepasst werden. Alternativ sind auch systematische Monitoringprogramme mit standardisierten Falldefinitionen (Surveillance) oder auch eine expositionsbezogene Fall-Kontroll-Studie mit einem geeignet gewählten Beobachtungsgebiet denkbar. In diesem Fall sollte epidemiologischer Sachverstand hinzugezogen, die Empfehlungen der Guten Epidemiologischen Praxis [7] beachtet und mit einer realistischen Kostenplanung (ggf. Mittel- 
einwerbung) begonnen werden. Das gestufte Vorgehen bei einer Clusteruntersuchung wird nach einem kurzen Exkurs über Ursachen des Auftretens von Clustern beschrieben.

\section{Exkurs: Erklärungsansätze für das Auftreten von zeitlich-räumlichen Clustern}

Eine geläufige Erklärung für Cluster ist das räumlich-zeitlich gehäufte Auftreten von Neuerkrankungen im Zusammenhang mit übertragbaren Krankheiten. Als Epidemie wird das für eine Region und einen Zeitraum festgestellte Auftreten einer ungewöhnlichen oder unerwartet hohen Zahl von Erkrankungen bezeichnet [8] - im hier gebrauchten Sinne ein Cluster. Von einer Endemie spricht man, wenn Erkrankungen in einer Region oder einem Land immer wieder auftreten, und mit Pandemie ist ein zeitlich gehäuftes Auftreten von Erkrankungen in der ganzen Welt oder in ganzen Erdteilen gemeint. Je nach Wechselbeziehungen zwischen den jeweiligen Erregern (agent) und den Infizierten (host) wird in Abhängigkeit von den Übertragungsprozessen und den hygienischen Bedingungen der jeweiligen Umgebung eine zeitlich-räumlich eng umgrenzte, eine endemische oder gar eine pandemische Krankheitsausbreitung zu erwarten sein. Dieses hängt gleichzeitig von der Expositionsstärke und von der Form und den Wegen der Übertragung sowie von der Empfindlichkeit (Immunität etc.) der Bevölkerung und von den Charakteristika des Erregers (Infektiosität, Inkubationszeit, Pathogenität, Virulenz, Stabilität etc.) ab. Bei Infektionen, die durch Parasiten übertragen werden, haben Krankheitsausbrüche z. B. dann eine höhere Wahrscheinlichkeit von räumlichen und zeitlichen Häufungen, wenn die Parasiten räumlich ungleich verteilt auftreten oder ihre Population in bestimmten Jahreszeiten größer (oder aktiver) ist.

Beispiele für räumlich-zeitliche Häufungen als Ausgangspunkt infektionsepidemiologischer Erklärungsansätze sind u. a. das Auftreten der Cholera in London 1854 (John Snow [9]: Kartierung der Fälle und Sperrung eines Trinkwasserbrunnens im geografischen Zentrum der Fälle), die
Aufklärung der Ursachen für den Ausbruch der Legionärskrankheit in Philadelphia 1976 (Ursachenabklärung für die Gruppe der Erkrankten: Klimaanlage/ Duschwasser) wie die Entdeckung der HIV-Verursachung [10] durch regional gehäuft aufgetretene Fälle einer seltenen Form der Lungenentzündung und des Kaposi-Sarkoms bei jungen Männern (Identifizierung des sexuellen Kontaktes der Fälle untereinander).

Die Ausgangssituation bei zeitlichräumlich eng zusammenliegenden beobachteten Erkrankungshäufungen infektiöser Genese ähnelt der im Umweltkontext diskutierten und zeigt $\mathrm{z}$. T. methodische und inhaltliche Überschneidungen. In der epidemiologischen Analyse einer möglichen infektiösen (Mit-)Verursachung von Leukämien im Kindesalter [11, 12] wird bei konstatierter räumlich-zeitlicher Clusterung der Fälle weiterhin kontrovers diskutiert, ob aus Wohnbiographien (Mutter/Kind) und aus dem Geburts- und Inzidenzdatum eine Assoziation zu möglichen Infektionsquellen erkennbar ist und welcher Risikobeitrag aus diskutierten Umweltbedingungen ( $\mathrm{z}$. B. Nähe zu kerntechnischen Anlagen, Hochspannungsleitungen, stark befahrenen Straßen) resultieren kann.

Als historisches Beispiel für ein anfangs als infektiös vermutetes und abschließend als anthropogen-umweltbedingt erkanntes räumlich eng umgrenztes Auftreten einer „Epidemie einer unbekannten Krankheit des Zentralnervensystems" in kleinen Dörfern an der Minamata Bucht kann das Minamata-Cluster aus dem Jahr 1956 in Japan gelten [13]. Im Zeitverlauf zunehmend, traten dort lokal gehäuft Erkrankungen mit sensorischen und motorischen Störungen in den distalen Bereichen der Arme und Beine, der Lippen, einer konzentrischen Einengung des Gesichtsfeldes, Funktionsstörungen des Kleinhirns (zerebelläre Ataxie), Bewegungsstörungen der Augen, Gleichgewichtsstörungen etc. und später Todesfälle und Fehlbildungen bei Neugeborenen auf. Die Personen wurden zur Unterbindung möglicher Infektionsketten anfangs stationär isoliert und ihre Häuser desinfiziert. Die Betroffenen waren, wie sich 1958 zeigte, mittelbar durch Methylquecksilber aus industriellen Einleitungen in die Bucht und direkt durch dauerhaft hohe Exposition über Fischkonsum aus lokalen Fängen vergiftet.

Aus der Arbeitsmedizin ist die in den 196oer-Jahren erkannte Überhäufung von Mesotheliomen bei Arbeitnehmern, die Asbest verarbeitet haben, ein Beispiel für die Identifizierung eines Stoffes mit kanzerogener Wirkung [14] auf der Grundlage von Einzelbeobachtungen. Obwohl die Latenzzeit zwischen einer hohen Exposition gegenüber luftgetragenen Mineralpartikeln und der Erkrankung mit mehr als 10 Jahren eingeschätzt wird, konnte die gemeinsame Ursache dieser Krebserkrankung schnell identifiziert werden. Da die Erkrankung ohne Exposition sehr selten ist und am stärksten diejenigen betraf, die im Arbeitsleben gegenüber Asbest hoch exponiert waren [15], war die Ursache vergleichsweise einfach zu identifizieren. Eine Zusammenfassung des Zugangs zu beobachteten arbeitsbezogenen Krankheitshäufungen ist $u$. a. bei Fleming etal. [16] zu finden.

Regionale Erkrankungshäufungen, die durch chemische oder physikalische Umweltbelastungen bedingt sind, können nur dann aufgeklärt werden, wenn die der Wirkung vorausgehenden Ursachen (Wirkungskette: Freisetzung der Noxe - Transport/Kontamination der Umweltmedien - Exposition über die orale, inhalative oder dermale Aufnahme - toxischer/kanzerogener Effekt) erkannt und in den $\mathrm{Zu}$ sammenhang des zeitlichen und regionalen Auftretens von Erkrankungsfällen (Inzidenz) gestellt werden. Dies setzt Wissen über die jeweiligen Noxen, ihren Transport und ihre Umwandlung in der Umwelt, über die Expositionspfade, die Expositionsstärke und die toxikologischen Wirkungsmechanismen (Dosis-Wirkungs-Beziehung, Variation in der Empfindlichkeit der Exponierten usw.) voraus. Einer zeitlich-räumlich eingrenzbaren Erhöhung des Erkrankungsrisikos muss eine räumlich begrenzt auftretende Erhöhung der Exposition zeitlich vorausgehen, um in Richtung einer ursachenbedingten Clusterbildung wirksam zu werden. Hierbei sind die auf die zeitliche Verteilung der Fälle Einfluss nehmenden Größen wie Akkumulation, Latenzzeit zwischen Exposition und Effekt sowie mögliche Wirkungsschwellen von Bedeutung. Tritt eine 
relevante Exposition für die Gesamtbevölkerung ab einem bestimmten Zeitpunkt auf oder verstärkt sich über die Zeit, so kann dies zu einer zeitlichen Häufung von Fällen ohne ausgeprägte räumliche Struktur führen. Es zeigt sich ggf. alleine eine zeitliche Häufung. Ist die Exposition mit anderen räumlich oder zeitlich variierenden Größen assoziiert (z. B. Sozialschicht, bestimmte Nutzergruppen für Produkte, regional ungleiche Markteinführung von Medikamenten/Produkten etc.), so kann sich in dieser Situation eine räumliche Heterogenität zeigen, ohne dass die Ursache selbst raumbezogen wäre.

Die Aufklärung von Clustern hat immer einen retrospektiven Charakter. Ein in die Vergangenheit gerichteter Ansatz der Ursachenklärung setzt eine möglichst vollzählige Datenlage zur Aufenthalts-, Wohn- bzw. Expositionsbiographie als Basis einer zeit- und raumbezogenen Expositionsabschätzung für die Fälle und die Bestimmung der Erwartungshäufigkeit für eine definierte Bezugspopulation voraus. Vor diesem Hintergrund wird deutlich, dass zeitlich-räumliche Inzidenzhäufungen eher identifiziert und gemeldet werden, wenn es sich um ein abgrenzbares bzw. definiertes Krankheitsbild handelt. Wenn die Ätiologie der berichteten Erkrankungen bekannt ist, wird die Frage nach möglichen Einflussfaktoren präziser zu stellen sein. Eine Häufung wird eher erkannt werden, wenn die Erkrankung allgemein selten oder in bestimmten, sonst selten betroffenen Alters- und Geschlechtsgruppen auftritt. Je früher ein Hinweis auf Häufungen von Erkrankungen erfolgt, desto eher kann mit der Abklärung möglicher Ursachen begonnen werden.

\section{Gestuftes Vorgehenskonzept}

Häufig sind es niedergelassene, individualmedizinisch tätige Ärzte/Ärztinnen, die über den wohnortnahen Patientenkontakt eine mögliche Krankheitshäufung in einer Gemeinde oder einem Wohngebiet (Cluster) feststellen. Die Wahrnehmung einer „ungewöhnlichen Häufung von Krankheitsfällen" erfolgt aber auch durch die Kommunikation innerhalb von Nachbarschaften, Dorfgemeinschaften oder in Kollegien von Betrieben bzw. in Schulen.

Tabelle 1

Unterscheidung der Ziele und Strategien bei Clusteruntersuchungen

\begin{tabular}{|c|c|c|}
\hline & $\begin{array}{l}\text { Untersuchungsstrategie: } \\
\text { Cluster - Quelle }\end{array}$ & $\begin{array}{l}\text { Untersuchungsstrategie: } \\
\text { Quelle/Exposition - Cluster }\end{array}$ \\
\hline "Cluster response" & $\begin{array}{l}\text { Bericht über (Krankheits-) } \\
\text { Cluster }\end{array}$ & $\begin{array}{l}\text { Beschwerden über mögliche } \\
\text { Gefahrenquelle }\end{array}$ \\
\hline "Cluster monitoring“ & $\begin{array}{l}\text { Frühzeitiges Aufdecken eines } \\
\text { Clusters } \\
\text { Follow-up eines Clusters }\end{array}$ & $\begin{array}{l}\text { Expositionsmonitoring } \\
\text { Nachbeobachtung einer } \\
\text { Exposition }\end{array}$ \\
\hline "Cluster research" & $\begin{array}{l}\text { Beschreibende Studien } \\
\text { Generelle Clusteruntersuchungen }\end{array}$ & $\begin{array}{l}\text { (kleinräumige) ökologische } \\
\text { Studien } \\
\text { Replikationsstudien um } \\
\text { Punktquellen } \\
\text { Studien an akuten Unfällen }\end{array}$ \\
\hline
\end{tabular}

Zur Überprüfung des Verdachtes einer Krankheitshäufung ist ein systematisches Vorgehen notwendig. Zur Initiierung einer solchen Untersuchung ist eine Information des Gesundheitsamtes oder des betriebsärztlichen Dienstes der erste Schritt. Hier können die weiteren Aktivitäten und Maßnahmen koordiniert und dokumentiert werden.

Wenn einer Gesundheitsbehörde plausible Informationen über regional auftretende Häufungen von bestimmten Krankheiten, Fehlbildungen oder Gesundheitsstörungen vorliegen, muss sie die notwendigen Schritte zur Klärung des Sachverhaltes einleiten und gegebenenfalls die zur Einordnung erforderlichen Daten selbst ermitteln. Ein gestuftes Vorgehen ermöglicht es den verantwortlichen Stellen, strukturiert und rational auf vorliegende Clusterhinweise zu reagieren, die angemessenen Schritte zur Klärung der Situation zu initiieren, die Öffentlichkeit und die parlamentarischen Gremien angemessen und zeitnah zu informieren. Falls erforderlich, können diese Stellen unverzüglich fachlich begründete und zur spezifischen Gefahrenabwehr notwendige expositionsmindernde Maßnahmen in die Wege leiten. Hierzu wird die nachfolgend beschriebene Verfahrensweise vorgeschlagen.

In den letzten Jahrzehnten wurden verschiedene Richtlinien dazu veröffentlicht, wie Anfragen zu einer möglichen Häufung von nicht-übertragbaren Erkrankungen aufzunehmen und weitergehend zu bearbeiten sind [17, 18, 19, 20, 21, 22, 23]. Allen gemeinsam ist ein stufenweises Vor- gehen ( $\bullet$ Tabelle 1 ), wobei die jeweils intensivierte nächste Bearbeitungsstufe von den Ergebnissen der vorangegangenen Bearbeitungsstufen abhängt. Die Centers for Disease Control $[6,24]$ veröffentlichten Empfehlungen für einen systematischen Ansatz, an denen sich die folgende Darstellung weitgehend orientiert. Solche Richtlinien sind flexibel zu verwenden und dabei an die Bedürfnisse im konkreten Einzelfall anzupassen.

\section{Erstkontakt}

Dem Erstkontakt mit einer den Verdacht äußernden Person kommt eine zentrale Bedeutung zu. Die Risikokommunikation beginnt in diesem Moment. Ein Erstkontakt kann schriftlich, per E-Mail oder telefonisch erfolgen. Im ersten Fall bietet sich ein Rückruf bei dem Meldenden an, wobei bereits vorab Zusatzinformationen (Verdacht bereits andernorts vorgebracht? Liegen Daten vor?) eingeholt werden können. Beim Telefonat müssen das Anliegen, der Informationsbedarf sowie mögliche Ängste und Befürchtungen ernst genommen werden. Oft können Verdachtsmomente für die Verursachung einer vermuteten/gesehenen Erkrankungshäufung, sofern der Ansprechpartner entsprechende epidemiologische bzw. umweltmedizinische Kompetenz besitzt, bereits angesprochen und ein mögliches weiteres Vorgehen skizziert werden.

In der Institution, die die Untersuchung übernommen hat, z. B. dem örtlichen Gesundheitsamt, sollte nach einem solchen Hinweis unmittelbar eine zuständige Per- 
son bestimmt werden, bei der alle Informationen zu dieser Clustermeldung zusammengeführt werden. Über diese Person wird der Kontakt zum Meldenden gehalten und die gesamte Untersuchung einschließlich der externen Kontakte dokumentiert. Eine zeitnahe Information der zugehörigen Pressestelle und übergeordneter Institutionen über den Anlass und das geplante Vorgehen kann unnötige Konflikte über „eine mangelnde Beteiligung und Kooperation“ vermeiden. Ist bekannt, dass vor Ort Bürgerinitiativen bereits aktiv sind oder bereits andere Stellen einbezogen wurden, sollte allen Beteiligten und Interessierten mitgeteilt werden, wer die Federführung im weiteren Verfahren übernimmt.

Man kann nicht davon ausgehen, dass die Information und Nachfrage ausschließlich an das zuständige Gesundheitsamt gegeben wird. Häufig werden verschiedene Institutionen (Umwelt- oder Sozialministerien, Landesgesundheitsämter, Ärztekammern, Krebsregister) zeitgleich informiert und parallel um Klärung gebeten. Hier gilt es, zeitnah die meldende Person an den primär zuständigen Ansprechpartner weiterzuleiten, der dann möglichst als einziger, koordinierender Ansprechpartner über das konkrete Vorgehen informieren sollte. Dies wird in der Regel das lokale Gesundheitsamt sein. Insofern sind „im Erstkontakt“, der als erste Stufe der systematischen Bearbeitung von Clusteranfragen anzusehen ist, die Anfragenden zunächst auf kompetente Stellen zu lenken. Es gilt insbesondere zu vermeiden, dass bereits im Vorfeld durch unterschiedliche Institutionen verschiedene, nicht abgestimmte Stellungnahmen, Situationseinschätzungen oder Maßnahmeempfehlungen abgegeben werden.

Im Erstkontakt kann bereits Information zur allgemeinen Häufigkeit der genannten Erkrankungsformen, ggf. zu bekannten Risiken und zu dem üblichen Vorgehen bei Clusteruntersuchungen gegeben werden. Hierbei kann, abgestimmt auf die Anfrage, bereits auf zu erwartende Probleme der Datenlage, deren Auswertung sowie der Interpretierbarkeit der Ergebnisse hingewiesen werden. Neben den gemeldeten Informationen und deren $\mathrm{Ab}$ gleich, ist beim Erstkontakt auch eine verbindliche Absprache zum weiteren Vorge- hen wichtig - häufig in Form der Ankündigung eines erneuten Anrufs, einer schriftlichen Antwort oder eines Gesprächstermins. Im Rahmen des Erstkontaktes wird unter Umständen bereits darum gebeten, Messdaten zur Umweltsituation neu zu erheben. Voreilige Messungen sollten in dieser frühen Untersuchungsstufe vermieden werden, da sie möglicherweise nicht zielgerichtet sind, später ggf. teure Mehrfachmessungen erzwingen und oftmals keine sachgerechte Interpretation zulassen. Falls es angezeigt erscheint, sollten Messungen systematisch vorbereitet und durchgeführt werden.

In Verdachtsmeldungen wird in Deutschland häufig über Cluster von Krebserkrankungen berichtet, es kann sich jedoch auch um zeitlich-räumliche Häufungen von chronischen Erkrankungen, Vergiftungen, Verletzungen, Fehlbildungen bei Neugeborenen oder Sterbefälle mit seltenen oder ungewöhnlichen ${ }^{1}$ Todesursachen handeln. Cluster können nur dann als solche identifiziert und verifiziert werden, wenn die Anzahl der beobachteten Fälle in der regionalen Bevölkerung oder in dem Betrieb, in der ein Cluster vermutet wird, der Anzahl der erwarteten Fälle in einer vergleichbaren Bevölkerungsgruppe oder der Allgemeinbevölkerung gegenübergestellt werden kann.

\section{Aufnahme der Daten zum Cluster- verdacht}

Zur Einordnung der Verdachtsmeldung über ein Cluster sollten im ersten Gespräch Einschätzungen und, soweit durch die meldende Person möglich, Informationen zu folgenden Punkten erhoben werden:

- Art der gehäuft auftretenden Symptomatik, Erkrankung oder der gesehenen Gesundheitsbeeinträchtigungen,

- Anzahl der betroffenen Personen (Fälle) und Größe der Bevölkerungsgruppe, zu der diese Personen gehören,

- Einschätzung der Alters- und Geschlechtsstruktur der Fälle,

\footnotetext{
${ }^{1}$ Hier ist ggf. im Einzelfall auch die Einbeziehung von Ermittlungsbehörden oder der Polizei angezeigt.
}

- Angaben zur räumlichen Ausdehnung des Clusters bzw. wahrgenommene regionale Ausbreitung der Auffälligkeit,

- zeitliche Angaben zum vermutlichen Beginn der Erkrankungshäufung, zur Dauer und zu gesehenen Veränderungen über die Zeit (Trends der Entwicklung),

- verdächtigte frühere oder aktuelle (Umwelt-)Exposition und, falls eine Verdachtsnennung möglich ist, auch eine Einschätzung der Expositionsdauer und -stärke,

- mögliche Begleitumstände/Veränderungen in der betroffenen Region.

Da Neuerkrankungsraten (Inzidenz) wie auch die krankheitsspezifische Prävalenz bzw. die Fehlbildungs- oder Sterberaten je nach Geschlecht und in Bezug auf das Lebensalter sehr unterschiedlich sein können, wird die Angabe einer „bloßen“ Anzahl von betroffenen Personen für eine Prüfung und Verifizierung eines Verdachts nicht ausreichend sein. Um einen korrekten Abgleich mit dem Erwartungswert der Allgemeinbevölkerung zu ermöglichen, müssen für die in die Clusterverdachtsprüfung einbezogenen Fälle (möglichst genaue) Angaben

- zum Alter (bei Erkrankungsbeginn bzw. Diagnosestellung),

- zum Geschlecht,

- zur Art der aufgetretenen Symptomatik, der Erkrankung oder der gesehenen Gesundheitsbeeinträchtigungen (falls bereits möglich in ICD-Kodierung),

- zur Anzahl der betroffenen Personen

(Summe der Fälle)

ermittelt und dokumentiert werden (siehe auch Ablaufdiagramm, • Abb. 1). Für die geografische Zuordnung jedes Falles werden Ortsangaben, am besten als Wohnadresse, benötigt. Jeder Fall, der zu dem Clusterverdacht beiträgt, muss eindeutig identifizierbar sein, um sicherzustellen, dass jeder Fall nur einmal in die weiteren Berechnungen eingeht.

Dies bedeutet nicht, dass in dieser Phase bereits eine Namensliste erstellt werden muss, sondern dass noch eine anonymisierende Identifikationsnummer ausreicht. Die Fallliste muss im loka- 


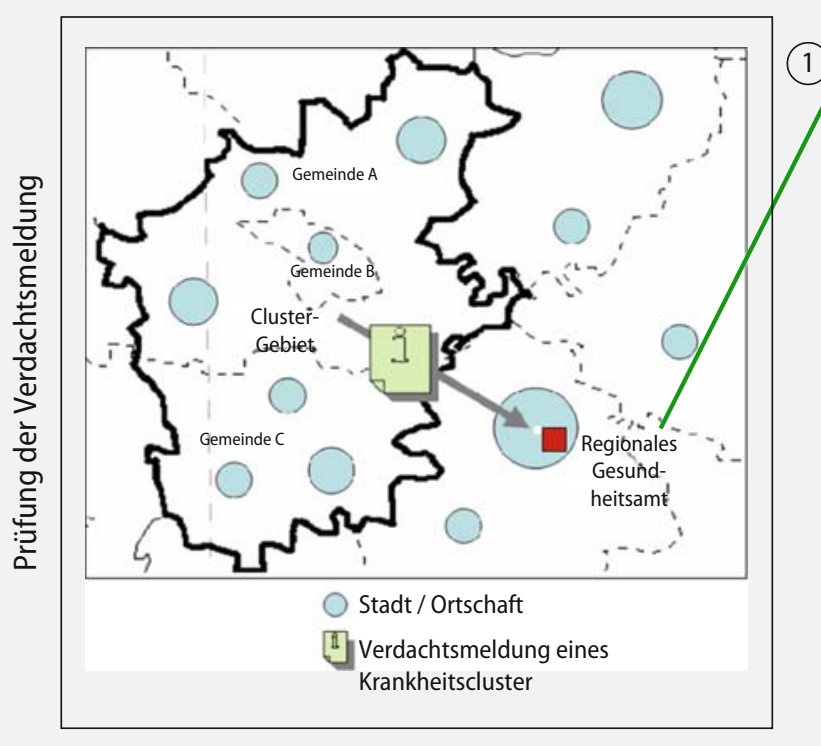

höher als der Erwartungswert: Epidemiologischer Anfangsverdacht

Kontaktaufnahme mit Melder zur Information und Absprache der weiteren Schritte zur Fallvalidierung/ Kontakt mit übergeordneter Stelle

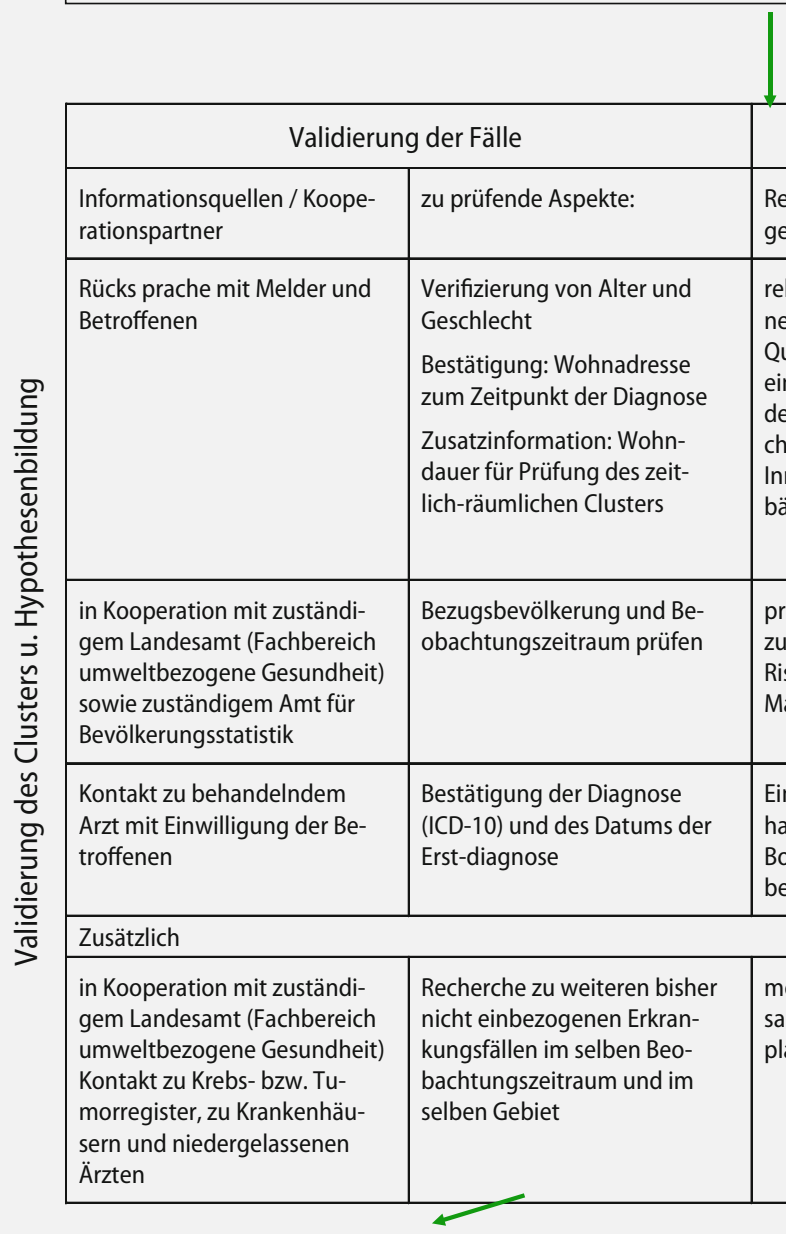

Verdacht erhärtet und Risikofaktor identifiziert: Minderungsmaßnahmen

(4)

bäude überprüfen)

prüfen, ob der Erkrankung zuzuordnende, bekannte, externe

Risikofaktoren (im relevanten

$\mathrm{Maß)}$ vorhanden sind

Einbeziehung möglicher vorhandener Messwerte (Wasser, berücksichtigen mögliche den Fällen gemeinsame Exposition am Arbeitsplatz recherchieren

Regionales Gesundheitsamt (rGA):

Erstkontakt mit Melder zur Erstellung einer Fallliste für das vermutete Krankheitscluster

Fallliste (Beispiel)
\begin{tabular}{|c|c|c|c|l|c|l|}
\hline $\begin{array}{l}\text { Identifikations } \\
\text {-Nr. des Falles }\end{array}$ & $\begin{array}{c}\text { Geschlech } \\
\mathrm{t}\end{array}$ & Geburtsjahr & Alter & Diagnose & $\begin{array}{l}\text { Datum der } \\
\text { Erstdiagnose } \\
\text { (Monat/Jahr) }\end{array}$ & $\begin{array}{l}\text { Wohnort zum Zeit- } \\
\text { punkt der } \\
\text { Diagnose }\end{array}$ \\
\hline 1 & $\mathrm{~m}$ & 1961 & 46 & Nieren-Ca & $11 / 2006$ & Gemeinde A \\
\hline 2 & $\mathrm{w}$ & 1967 & 40 & Nierenzellkrebs & $01 / 2007$ & Gemeinde B \\
\hline 3 & $\mathrm{~m}$ & 1959 & 48 & NCC & $05 / 2007$ & Gemeinde C \\
\hline 4 & $\mathrm{~m}$ & 1955 & 52 & Nierenkarzinom & $12 / 2006$ & Gemeinde B \\
\hline$(\ldots)$ & & & & & & \\
\hline
\end{tabular}

(2)

rGA: Orientierende Evaluation

beobachtete Fälle (Fallliste)

vs.

erwartete Fälle (z.B. Krebsregister)

(3)

3

geringer als
Erwartungswert:
Komschluss und
Kommunikation mit
der Öffentlichkeit/

Hypothesenbildung zu möglicher Ursache

Recherche möglicher Belastun- $\quad$ Informationsquellen / Kooperagen: $\quad$ tionspartner

relevante (Umwelt-) Expositio- $\quad$ Literaturrecherche und Datennen recherchieren (vermutete banken (z.B. NoxeninformatiQuelle der Exposition wie z.B. onssystem (NIS, LANUV NRW), eine nahegelegene Sondermüll- $\quad$ BfR, Gefahrstoffinformationsdeponie/-verbrennungsanlage, system GESTIS, Informationschem. Produktionsstätte oder $\quad$ system gefährli-

Innenausstattung in Schulge- che/umweltrelevante Stoffe

(IGS), DIMDI, etc.

Landesamt für Umwelt und/oder Gesundheit

Landesamt für Umwelt und/oder Gesundheit

Bei Hinweis auf krankheitsspezifisch relevante oder bekannte Produktionsstätten im Umkreis des Clustergebietes

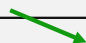

Hypothesen zu möglichen Ursachen verifizieren: Epidemiologisch-analytische Studie 


\section{Empfehlung des Robert Koch-Instituts}

Tabelle 2

Fallliste ${ }^{a}$ (artifizielles Beispiel)

\begin{tabular}{|c|c|c|c|c|c|c|}
\hline $\begin{array}{l}\text { Identifikations-Nr. } \\
\text { des Falles }\end{array}$ & Geschlecht & Geburtsjahr & $\begin{array}{l}\text { Alter bei } \\
\text { Diagnose- } \\
\text { stellung }\end{array}$ & Diagnose & $\begin{array}{l}\text { Diagnose- } \\
\text { datum } \\
\text { (Monat/Jahr) }\end{array}$ & $\begin{array}{l}\text { Wohnort zum } \\
\text { Zeitpunkt der } \\
\text { Diagnose }\end{array}$ \\
\hline 1 & m & 1961 & 46 & Nieren-Ca & $11 / 2006$ & Gemeinde A \\
\hline 2 & w & 1967 & 40 & Nierenzellkrebs & $01 / 2007$ & Gemeinde B \\
\hline 3 & m & 1959 & 48 & NCC & $05 / 2007$ & Gemeinde C \\
\hline 4 & m & 1955 & 52 & Nierenkarzinom & $12 / 2006$ & Gemeinde B \\
\hline \multicolumn{7}{|l|}{$(\ldots)$} \\
\hline
\end{tabular}

len Gesundheitsamt sicher verwahrt werden, in der öffentlichen Kommunikation sollten wegen der Gefahr einer Identifizierung (ggf. Stigmatisierung) keine detaillierten Angaben zum Alter, zum Geschlecht und der Wohnregion/-adresse der Betroffenengruppe gemacht werden. Eine zusammenfassende, die Fallgruppe insgesamt beschreibende Aussage ist für die Öffentlichkeitsarbeit ausreichend und angemessen.

Bestehende Datenschutzrichtlinien und persönliche Wünsche der Betroffenen, in welchem Maße Daten und Informationen zu ihrer Person und Erkrankung in die weitere Untersuchung eingehen, müssen vor dem Hintergrund des informationellen Selbstbestimmungsrechtes der Betroffenen berücksichtigt werden. Für Untersuchungen im Geltungsbereich des Gesetzes zur Verhütung und Bekämpfung von Infektionskrankheiten beim Menschen (Infektionsschutzgesetz, IfSG) [25] gelten für meldepflichtige Erkrankungen ggf. Bestimmungen, die die Individualrechte einschränken.

Für eine Überprüfung einer „Clustermeldung" sollten im Gesundheitsamt oder vom meldenden Arzt Parameter gemäß - Tabelle 2 in einer anonymen Fallliste zusammengestellt werden. Diese stellt die Basis für die spätere Kommunikation, z. B. mit dem Landesgesundheitsamt, mit dem Krebsregister oder mit einer anderen Institution dar und wird auch der Ausgangspunkt einer orientierenden Evaluierung des Clusterverdachtes sein. Bei der Erstaufnahme eines Clusterverdachts sollte von den Datengebern erfragt werden, ob und wie verlässlich die Fallliste als vollzählig einzustufen ist und wo ggf. wei- tere Daten zu entsprechenden Diagnosen zu erheben wären.

Sofern es sich bei der Clusterverdachtsmeldung um eine kleinräumige Häufung handelt, die sich auf benannte oder vermutete Risikoquellen bezieht, muss ggf. additiv der Wohnort mit Adressangabe als Basis für distanzabhängige Betrachtungen erhoben werden. Hierbei ist die datenschutzrechtliche Notwendigkeit des Einholens einer Einwilligungserklärung zu prüfen.

Die möglichst weitgehende Vervollständigung der Fallliste ist ein schwieriger und zeitaufwendiger Arbeitsschritt, der daher ggf. parallel zu den weiteren Schritten durchzuführen ist. Das Gesundheitsamt ist hierbei auf eine enge Kooperation mit den behandelnden Ärzten/Ärztinnen der Region angewiesen. Ziel des Kontaktes zur niedergelassenen Ärzteschaft und ggf. im Einzugsbereich liegenden Diagnoseund Therapieeinrichtungen muss es sein, (a) die Diagnosen der Personen in einem vermuteten Cluster zu sichern und (b) einen hohen Grad der Vollzähligkeit der Fallliste zu erreichen. Durch die Diagnosesicherung muss überprüft werden, ob möglicherweise eine Zusammenstellung heterogener Krankheitsbezeichnungen/ Symptome/Lokalisationen ähnlicher Erkrankungen zu einer scheinbaren Häufung in der Fallliste führen kann. Durch die Vervollständigung der Fallliste muss versucht werden, die gesundheitliche Lage der Bevölkerung bezüglich der Häufigkeit der Erkrankung angemessen zu beschreiben. Für die Untersuchung von Clusterverdachtsmeldungen bietet sich dabei die Einbeziehung der bevölkerungsbezogenen (epidemiologischen) Krebsregister oder ggf. anderer Krankheitsregister oder Fehlbildungsregister an. Sie können geeignete Referenzzahlen, etwa landkreisspezifische Inzidenzen, zur Verfügung stellen oder auch zur Vervollständigung der Fallangaben beitragen, indem sie geeignete Daten, oder auch Information über Datenquellen und -zugänge mitteilen. Allerdings sind gerade bei kleinräumigen Untersuchungen die Analysemöglichkeiten der Register häufig in unterschiedlicher Weise aufgrund der regionalen Auflösung der vorliegenden epidemiologischen Daten und auch aufgrund der einer Datenübermittlung zugrunde liegenden gesetzlichen Bestimmungen limitiert.

Jeder Kontakt mit meldenden Personen, einschließlich Ärzten/Ärztinnen, sollte nachvollziehbar dokumentiert werden. Es wird dringend empfohlen, ein Ablaufprotokoll mit Datumserfassung über alle Aktivitäten und Anforderungen an beteiligte Institutionen, Ärzte/Ärztinnen und Kliniken anzulegen, um ggf. eine kritische, auf Zeitabläufe gerichtete Diskussion bestehen zu können. Wenn der Erstkontakt und die Falldokumentation Hinweise darauf liefern, dass eine weiterführende Untersuchung notwendig ist, sollte sich eine „Vorläufige Evaluierung“ (der Schritt 2 bei Clusteruntersuchungen) anschließen. Diese Notwendigkeit kann sich entweder aus der geschilderten/belegten Fallhäufung, aus einer verdächtigten Exposition im Sinne einer ggf. notwendigen Gefahrenabwehr oder aus einer (beginnenden) öffentlichen Beunruhigung ergeben. Über die Vorgehensweise, die Methodik und die Ergebnisse dieses Arbeitsschrittes sollten alle Beteiligten informiert werden. 


\section{Einordnung der Beobachtungen: vorläufige Evaluierung}

Ist die Liste der Verdachtsfälle zusammengestellt, wird diese mit der erwarteten Anzahl von Erkrankungsfällen in der Allgemeinbevölkerung in der Region verglichen. Die Daten des Erstkontaktes, evtl. ergänzt um vorliegende Daten aus anderen Quellen, werden für eine vorläufige Berechnung der beobachteten und der erwarteten Häufigkeit herangezogen, um das Vorliegen einer Fallhäufung einzuschätzen. Dabei sind zunächst einige Festlegungen zu treffen und zu dokumentieren:

- Die geografische Abgrenzung der Untersuchungsregion: In der Regel wird man versuchen, auf bestehende regionale Gemeinde- oder Stadtteildefinitionen zurückzugreifen. Bei kleinräumigen Anfragen, die konkret einzelne Straßenzüge benennen, ist dieser Bereich entsprechend abzubilden, sodass auch Differenzierungen innerhalb der Untersuchungsregion bereits bei der vorläufigen Evaluation möglich sind.

- Die Festlegung der Beobachtungsperiode bzw. retrospektiven Beobachtungsdauer: Die Festlegung richtet sich zum einen nach den Erkrankungszeiträumen der genannten Fälle, anderseits nach der Möglichkeit, später die Fälle zu validieren bzw. Registerdaten heranziehen zu können. Je kleiner das Untersuchungsgebiet, desto länger muss die Beobachtungsdauer sein, um zu ausreichenden Fallwie Nennerangaben zu kommen. Ebenso wie bei der Untersuchungsregion bietet sich eine Unterteilung der gesamten Beobachtungsperiode etwa in 5- oder 10-Jahres-Teilperioden an. Je länger der Zeitraum zurückreicht, desto ungenauer werden Fall- wie Nennerangaben. Eine Betrachtung von mehr als 20 Jahren ist nicht zu empfehlen. Gegebenenfalls muss die Fallliste gemäß der festgelegten Beobachtungsperiode vervollständigt werden.

- Fallabgrenzung: Bestimmung, welche der vorläufigen „Fälle“ gerade bei unspezifischen Fallaufstellungen in die Analyse aufgenommen werden (an-

Tabelle 3

Internet-Adressen der nationalen Krebsregister

\begin{tabular}{ll} 
Bundesland & Internet-Adresse \\
\hline Baden-Württemberg & Im Aufbau \\
\hline Bayern & http://www.ekr.med.uni-erlangen.de/ \\
\hline $\begin{array}{l}\text { Berlin, Brandenburg, } \\
\text { Mecklenburg-Vorpommern, } \\
\text { Sachsen, Sachsen-Anhalt, Thüringen }\end{array}$ & http://www.berlin.de/gkr/ \\
\hline Bremen & http://www.krebsregister.bremen.de/ \\
\hline Hamburg & http://www.krebsregister.hamburg.de \\
\hline Hessen & http://www.laekh.de (>Krebsregister) \\
\hline Niedersachsen & http://www.krebsregister-niedersachsen.de/ \\
\hline Nordrhein-Westfalen & http://www.krebsregister.nrw.de/ \\
\hline Rheinland-Pfalz & $\mathrm{http://krebsregister-rheinland-pfalz.de}$ \\
\hline Saarland & $\mathrm{http://www.krebsregister.saarland.de/}$ \\
\hline Schleswig-Holstein & $\mathrm{http://www.krebsregister-sh.de/}$ \\
\hline
\end{tabular}

hand der Angaben zur Diagnose der gemeldeten Fälle). Diese Abgrenzung impliziert noch keine Fallüberprüfung bzw. -validierung.

- Festlegung der Bezugspopulation: In der Regel ergibt sich diese unmittelbar aus den zugrunde liegenden Abgrenzungen der Untersuchungsregion und der Beobachtungsperiode (ggf. eingeschränkt auf bestimmte Altersgruppen o. Ä.). Die benötigten Nennerangaben sind dann von den Meldeämtern zur Verfügung zu stellen. Bei einer Untersuchungsperiode über längere Zeiträume muss bei der Schätzung der zu erwartenden Fälle ggf. die demografische Entwicklung der Bezugspopulation berücksichtigt werden (Alterung, Mobilität). Clusterverdachtsmeldungen, die sich nicht auf die Wohnbevölkerung beziehen (z. B. mögliche Häufung auf einem Campingplatz oder bei Besuchern einer Veranstaltung), werden sich zunächst auf Angaben des Erstkontaktes und ggf. weitere Einschätzungen zur Fallzahl und zur möglicherweise exponierten Bevölkerungsgruppe stützen müssen.

Bei Krebsclusteranfragen wird man dabei entsprechende Inzidenzraten der epidemiologischen Krebsregister heranziehen, wenn verfügbar, die des zuständigen Landeskrebsregisters für den übergeordneten
Bezirk oder Landkreis. Zeigen die Maßzahlen über die Jahre eine Veränderung (z. B. Abnahme der Magenkrebssterblichkeit), ist dies - abhängig von der Untersuchungsperiode - zu berücksichtigen. Die Basisdaten (Inzidenz, Mortalität) zur Berechnung der erwarteten Anzahl von Erkrankungs- oder Sterbefällen können bei einer Verdachtsmeldung zu Krebserkrankungen über die Datenbanken der epidemiologischen Krebsregister der Bundesländer bzw. für bundesweite Aussagen über die Dachdokumentation Krebs des Robert Koch-Institutes abgerufen werden. Die Datenbanken sind online über den jeweiligen Internetauftritt zugänglich. Ein Kontakt kann jedoch auch über die Landesgesundheitsämter oder entsprechende Landeseinrichtungen vermittelt werden. Die interaktive Datenbank der deutschlandweiten Dachdokumentation Krebs des Robert Koch-Institutes ist über den Pfad: http://www.rki.de/gbe/krebs und dann über die Menüpunkte Dachdokumentation Krebs - Datenbankabfragen Neuerkrankungen zu erreichen. Die Internetadressen der deutschen Krebsregister sind in $\bullet$ Tabelle 3 verzeichnet.

Das Deutsche Kinderkrebsregister ist über eine eigene Website zu erreichen: http://www.kinderkrebsregister.de. Das umfassendste deutsche Register zur Dokumentation von angeborenen Fehlbildungen befindet sich derzeit an der Kinderklinik des Universitätsklinikums in 
Tabelle 4

Fallunterscheidung für Handlungsoptionen nach Durchführung der ersten Clusterevaluation: Entscheidungsmatrix nach Ende der Clusteruntersuchung nach Quaetert [28]

\begin{tabular}{|lll}
\hline & Keine besondere Exposition & Besondere Exposition \\
\hline $\begin{array}{l}\text { Kein statistisch } \\
\text { erkennbares Cluster }\end{array}$ & $\begin{array}{l}\text { Zusammenfassender Bericht } \\
\text { (z. B. scheinbares Cluster nach Standardisierung nicht } \\
\text { erkennbar) }\end{array}$ & $\begin{array}{l}\text { Beseitigung/Verminderung } \\
\text { Überprüfung auf alternative } \\
\text { Gesundheitsindikatoren } \\
\text { (Zusammenhang mit Exposition belegt) }\end{array}$ \\
\hline $\begin{array}{l}\text { Statistische Hinweise } \\
\text { auf ein Cluster }\end{array}$ & $\begin{array}{l}\text { Nachbeobachtung: } \\
\text { „Post-Alarm-Monitoring“ } \\
\text { (ansonsten: ,Zufallsbedingtes“ Cluster) }\end{array}$ & $\begin{array}{l}\text { Durchführbarkeit von ätiologisch-epidemiologischen Studien } \\
\text { überprüfen }\end{array}$ \\
\hline
\end{tabular}

Mainz: http://www.mainzermodell.de/ content/Sitemap.46.o.html.

Für andere chronische Erkrankungen muss zur Ermittlung der erwarteten Anzahl von Fällen die Prävalenz in der Allgemeinbevölkerung herangezogen werden. Hier kann jedoch noch nicht auf öffentlich zugängliche Datenbanksysteme zurückgegriffen werden. Für die Überprüfung solcher Cluster-Verdachtsfälle sollte nach der Erstellung der Fallliste eine $\mathrm{Zu}$ sammenarbeit mit der jeweiligen Landesbehörde gesucht werden, da dort ggf. Daten aus der landesbezogenen Gesundheitsberichterstattung gehalten werden. Auch zu den nach IfSG [25] meldepflichtigen Infektionserkrankungen und zu der Anzahl von Krankenhausfällen durch Vergiftungen liegen in den Landesbehörden Daten vor. Über die Anzahl von Vergiftungsfällen können auch die Vergiftungsinformationszentralen Auskunft geben. Sind keine Prävalenzdaten aus der Gesundheitsberichterstattung verfügbar, können ggf. Prävalenzdaten epidemiologischer Studien aus der Region selbst oder aus strukturell vergleichbaren Regionen einen Anhaltspunkt bieten [26].

Die zweite Datenbasis, die für die Berechnungen herangezogen werden muss, ist die Bevölkerungszahl in dem Untersuchungsgebiet des Clusters. Hierzu muss das Gesundheitsamt, das die Clusterüberprüfung durchführt, die Einwohnerzahlen zum entsprechenden Untersuchungszeitraum von dem oder den jeweiligen Einwohnermeldeämtern für die Untersuchung anfordern. Vorab müssen das geografische Gebiet (Gemeinde, Orte) und der Zeitraum (Diagnosejahr des ersten Falls, Diagnosejahr des letzten Falls) des Clusters festgelegt werden. Hierbei ist ggf. eine Erweiterung des Zeitraumes der Betrachtung um Zeiten vor und nach dem vermuteten Cluster zu erwägen.

Mit diesen Vorgaben lassen sich übliche epidemiologische Maßzahlen (Inzidenzen, Prävalenzen u. Ä.) berechnen und alters- und geschlechtsstandardisiert mit den unter „normalen“ Umständen zu erwarteten Fallzahlen vergleichen. Als „normal“ wird angesehen, dass (a) in allen betrachteten Regionen und Zeitintervallen ein gleiches Grundrisiko für definierte Erkrankungen vorliegt und (b) dass die beobachtete Anzahl von Erkrankungen jeweils Variation um die Grundrate aufweisen wird. Die Bestimmung der erwarteten Anzahl von Erkrankungen beinhaltet für jede Alters- und Geschlechtsgruppe eine Multiplikation der normalerweise gesehenen Raten mit der Anzahl der Personen in der betrachteten Bevölkerung. Aus der Addition dieser Einzelwerte ergibt sich die bei der gegebenen Bevölkerungsstruktur zu erwartende Gesamtzahl von Fällen unter der Annahme einer in der Gesamtbevölkerung und der betrachteten Bevölkerungsgruppe gleichen Risikoverteilung. Liegen die beobachteten Fallzahlen über den erwarteten Fallzahlen, so deutet dies auf das Vorliegen einer regional-zeitlichen Risikoerhöhung hin. Liegt die Zahl der Beobachtungen so weit über der Erwartung, dass dies nicht mehr mit der zu erwartenden Variation (Jahrzu-Jahr-Schwankungen, insgesamt zu sehende regionale Schwankungsbreiten, Stichprobenfehler) zu erklären ist, so wird das Ergebnis als statistisch signifikant einzustufen sein. Bei der Beurteilung von statistischer Signifikanz muss aber immer auch berücksichtigt werden, dass einerseits die relativen Abweichungen bei kleineren Bevölkerungsgruppen größer sein müssen, um die Signifikanzschwelle $\mathrm{zu}$ überschreiten, dass aber andererseits bei kleineren Fallzahlen auch die relative Variationsbreite über die Zeit und die Regionen vergleichbar höher sein wird.

Sollte sich der Verdacht durch den Vergleich mit dem Erwartungswert nicht erhärten, müssen mit den Meldenden, der Kommune und den Betroffenen (bzw. ihren Vertretungen) der Befund und die gewählte Methode der Verdachtsüberprüfung erläutert werden. Dies kann sowohl in einem Gespräch oder einer öffentlichen Veranstaltung erfolgen. Eine kurze schriftliche Dokumentation sollte den Ablauf der Recherche, die wesentlichen Daten, die Methoden und die Ergebnisse zumindest in den Kernpunkten beschreiben. Eine Pressemitteilung und eine Information an andere anfangs oder im Prozess beteiligte Institutionen und Behörden gehören zur notwendigen Risikokommunikation. Allgemeine Empfehlungen zur Risikokommunikation wurden vom Deutschen Kinderkrebsregister entwickelt und veröffentlicht [27]. Um auf möglicherweise weiterhin bestehende Unsicherheiten einzugehen, kann mit dem lokalen Gesundheitsamt eine erneute Überprüfung der Inzidenzrate für ein festzulegendes Zeitintervall vereinbart werden. Denkbar ist jedoch auch, dass sich aufgrund von Merkmalen und Eigenschaften der Fälle ein Verdacht auf eine bestimmte Exposition ergibt, die nicht umweltbezogen sein muss, die aber eine ätiologisch orientierte Studie oder Nachverfolgung rechtfertigt. Die beschriebenen Alternativen sind in - Tabelle 4 zusammenfassend dargestellt. 


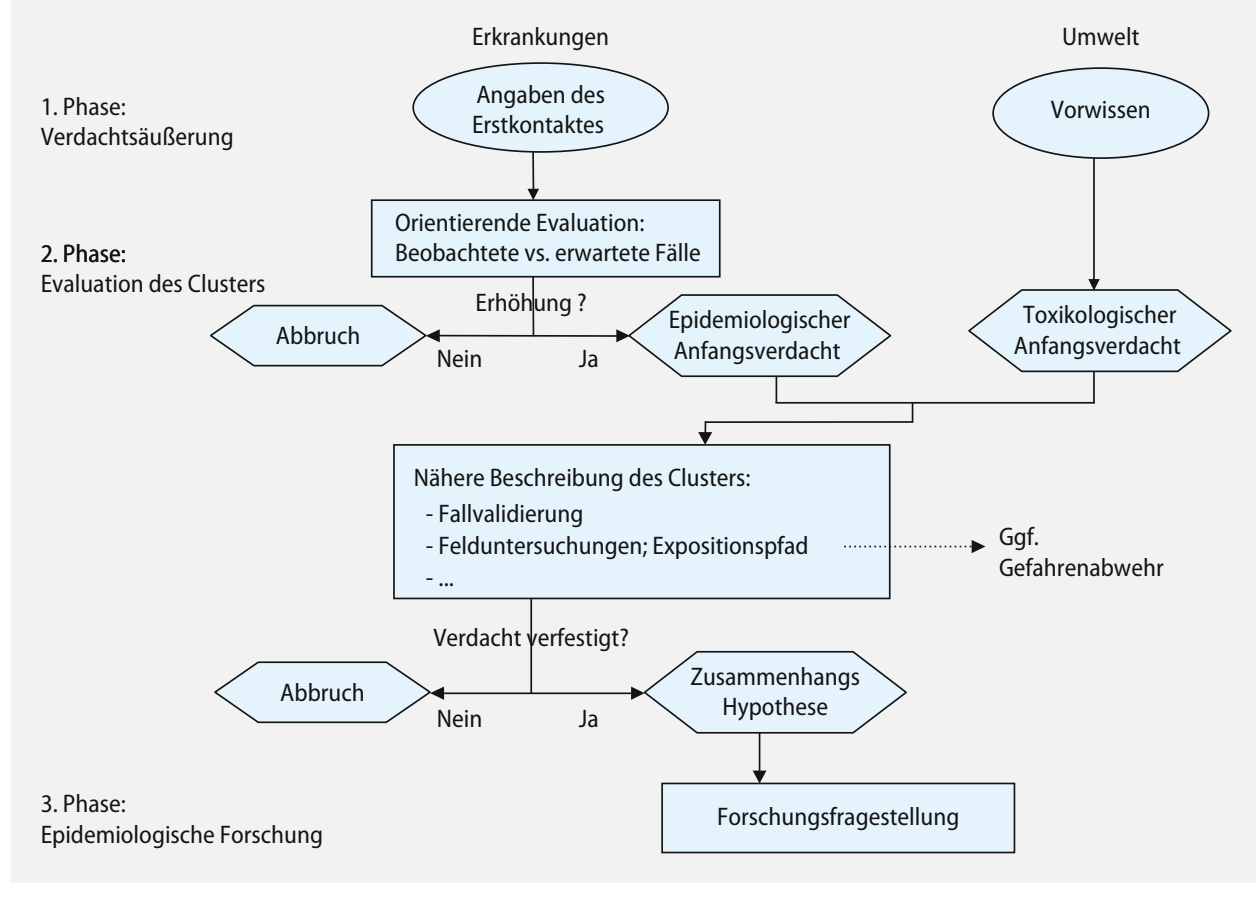

Abb. $2<$ Zusammenfassende Skizze der Phasen einer Clusterabklärung

\section{Vorarbeiten zu einer vertieften Clusterabklärung}

Sollte sich durch den Vergleich mit dem Erwartungswert in der Allgemeinbevölkerung der Anfangsverdacht einer Krankheitshäufung bestätigen (siehe - Abb. 2), sollten in der 2. Phase der Clusterabklärung übergeordnete Landesbehörden informiert werden und eine Fallvalidierung in Zusammenarbeit mit den Betroffenen und den behandelnden Ärzten/Ärztinnen eingeleitet werden. Die Landesgesundheitsämter bzw. obersten Landesbehörden sind in diese Validierung einzubeziehen. Spätestens zu diesem Zeitpunkt sollte auch die Frage nach der Koordination und nach der Finanzierung der weiteren Schritte erfolgen. $\mathrm{Ob}$ und ggf. welche Institution die notwendigen statistisch-epidemiologischen Arbeitsschritte durchführen kann, ob und ggf. wie eine Auftragsvergabe an ein externes (universitäres) Institut erfolgen muss, ist zu klären.

Die Entscheidung, an die vorläufige Evaluation eine aufwändige Clusteruntersuchung im engeren Sinne anzusetzen, sollte nicht allein von der „statistischen Signifikanz" einer Fallzahlüberhöhung gegenüber der Erwartung abhängen, sondern auch von der absoluten Fallzahl und zudem von Überlegungen zur Plausibilität eines möglichen Zusammen- hangs mit der vermuteten Exposition. Die Vermeidbarkeit der Exposition sowie der Kenntnisstand zu der die Häufung betreffenden Erkrankung sind zu berücksichtigen.

Daneben ist aber auch das öffentliche Interesse entscheidend. Dies kann dazu führen, dass auch ohne Hinweis auf eine tatsächliche Fallzahlerhöhung allein aufgrund einer weit verbreiteten Beunruhigung eine Clusterabklärung fortgesetzt wird [29]. Etwas konkreter als die CDCRichtlinien, über die eine Fortsetzung insbesondere dann begründbar ist, wenn die vorläufige Evaluierung eine Überhöhung nachgewiesen hat oder aber medizinische Plausibilität bzw. ein hohes öffentliches Aufklärungsinteresse vorliegt, sind die Richtlinien des Washington State Department [22], die folgende Kriterien vorschlagen:

- mindestens 3 gleichartige Fälle,

- und vermutliche Überhöhung,

- und eines der folgenden Kriterien:

- bei guter Kenntnis zur Ätiologie der Krankheit: Die Exposition gegenüber einem ursächlichen Wirkstoff scheint möglich.

- Die in Frage stehende Exposition war in der Vergangenheit schon einmal mit der berichteten Lage assoziiert.

- Die Ätiologie der beobachteten Erkrankung ist unbekannt, in dem betrachteten Fall existieren jedoch unübliche oder unklare Expositionsbedingungen.

- Die betrachtete Erkrankung tritt extrem selten auf.

Es wird vorgeschlagen, die Aussage einer vermutlichen Überhöhung dabei auf einem einseitig formulierten Test $\mathrm{zu}$ basieren mit einer nominellen Irrtumswahrscheinlichkeit von höchstens $5 \%$.

\section{Exkurs: Gültigkeit der nominellen Irrtumswahrscheinlichkeiten}

Die Betonung auf „nominiell“ bei der Irrtumswahrscheinlichkeit weist darauf hin, dass es sich bei klassischen Clusteruntersuchungen eben nicht um wohl geplante Studien mit vorab definierten Ziel- und Bezugsgrößen handelt, sondern um beobachtete Auffälligkeiten, die erst im Nachhinein „abgetestet“ werden.

Während in einer vorab definierten und nachfolgend beobachteten Region eine als zufällig einzustufende Häufung eben nur mit der vorgegebenen Irrtumswahrscheinlichkeit aufträte, ist allein aus Gründen von normalen Zufallsschwankungen das Auftreten von irgendeiner (nominell statistisch) auffälligen Region in einem größeren Gesamtgebiet (z. B. Bundesland) praktisch mit Sicherheit zu erwarten: 
Angenommen, in allen der rund 12.00o Gemeinden der Bundesrepublik Deutschland bestünde das gleiche Risiko für eine bestimmte Erkrankung: In dieser Situation würde man wegen der Vielzahl von in der Regel nicht bekannten Einflussgrößen variierende Erkrankungsraten erwarten. Wenn man nun für jede Gemeinde einzeln testen würde, ob eine statistisch signifikante Risikoerhöhung vorliegt, so würde bei einer üblichen Irrtumswahrscheinlichkeit von $5 \%$ für rund 600 der Gemeinden ein „falscher Alarm“ ausgelöst werden, obwohl de facto kein erhöhtes Risiko vorläge.

Das gleiche Problem ergibt sich, wenn eine hohe Zahl von Zeitintervallen bezüglich der Auftretenshäufigkeit von Erkrankungen betrachtet wird. Die Wahrscheinlichkeit, dass einzelne Zeitfenster von der Grundrate abweichen, steigt mit der Anzahl der betrachteten Intervalle. Wenn Regionaldaten zudem zeitlich geschichtet berichtet werden, verschärft sich dieses Problem nochmals, da die Anzahl der Vergleiche (a) über die betrachteten Zeitintervalle und (b) über die untersuchten Regionen zu einer Multiplikation der Anzahl durchgeführter Tests führt.

Die nominelle Irrtumswahrscheinlichkeit bezieht sich auf einen Einzelvergleich, während bei Clusteranfragen in der Regel implizit vorher mehrere Vergleiche durchgeführt wurden und erst die scheinbar auffälligste Erhöhung "getestet" wird. Als „texanische Scharfschützen“ bezeichnet man in der Epidemiologie ironisch solche Wissenschaftler, die erst in einer großen Datenmenge nach Auffälligkeiten suchen (d. h. übertragen: „das Ziel im Loch anfertigen"), um dann formal „auf Signifikanz zu testen“ (übertragen: „Schießen und mit Sicherheit treffen"). Bei dieser impliziten „Suche“, die sich einer genauen statistischen Kontrolle entzieht, treten sowohl das Problem der Optimierung zwischen Fallangaben und Bezugspopulation (Zähler-/Nennergrößen) auf als auch das Problem des impliziten „multiplen Testens“: In solchen Situationen sind vorab diverse alternative regionale Bezugsgrößen, Zeitintervalle und/oder auch Diagnosegruppen betrachtet worden, aber nur die scheinbar auffälligste Erhöhung führt möglicherweise zu einer Clusteranfrage.
Bei seltenen Erkrankungen kommt erschwerend hinzu, dass extrem hohe beobachtete Erkrankungs- oder Mortalitätsraten mit größerer Wahrscheinlichkeit in kleineren Gemeinden beobachtet werden. Das heißt, je kleiner die Bevölkerung in einer betrachteten Gebietseinheit ist, umso größer ist die Wahrscheinlichkeit, extreme Raten zu finden. Bei einer mittleren Inzidenz von 1/100.000 in einer Gemeinde mit 5000 Einwohnern genügen vier Erkrankungsfälle innerhalb von 10 Jahren, um ein signifikant erhöhtes relatives Risiko von $8 \mathrm{zu}$ erhalten [30]. In diesen Situationen ist eine Differenzierung zwischen einer ursachenvermittelten und einer variationsbedingten Erhöhung mit epidemiologischen Instrumenten kaum zu leisten. Eine nähere Clusteruntersuchung in Richtung ätiologischer Forschung auf Basis von nur 4 Fällen ist praktisch selten Erfolg versprechend.

Die verwendeten „Inferenzstatistiken“ und insbesondere das angegebene Niveau („Irrtumswahrscheinlichkeit“ erster Art) dürfen aus den o. g. Gründen daher nur deskriptiv verstanden werden.

Von einer deutlichen statistischen Auffälligkeit sollte bei der näheren Clusteruntersuchung erst auf Basis eines nominellen $1 \%$-Niveaus gesprochen werden. Währenddessen beschreiben Ergebnisse zum nominellen 5\%-Niveau, die oben als notwendig für den Übergang zur vertiefenden Evaluierung angegeben sind, eine statistische Auffälligkeit, die durchaus aufgrund von multiplen Vergleichen und explorativen Analysen variationsbedingt entstanden sein kann, aber dennoch aufgrund einer möglichen inhaltlichen Relevanz näher betrachtet oder nachbeobachtet werden sollte.

Eine Aufgabe der orientierenden Evaluation ist es, soweit möglich, (a) die explorative Abgrenzung des Clusterproblems von der zu erwartenden Variation (über Zeitintervalle oder Regionen) und (b) die fachliche Einordnung im Rahmen der Analyse und Bewertung möglicher Ursachen zu erreichen. Treten Häufungen von Erkrankungen in direkt benachbarten Regionen in enger zeitlicher Übereinstimmung auf, so hat die Clusterinformation eine höhere Bedeutung. Da übliche statistische Grundannahmen nur eingeschränkt gelten, sollte die Beurteilung der
Relevanz eines mutmaßlichen Clusters (Stufe: vertiefende Evaluation) entsprechend weniger auf Statistiken, sondern stärker auch auf pathophysiologisch orientierten Kriterien basieren.

\section{Clusterabklärung bei Annahme einer umweltbedingten Ursache}

Wenn zusammen mit der Clusterverdachtsmeldung eine oder mehrere Umweltquellen als Verursacher benannt werden, sollten Prüfung möglicher umweltbezogener Gesundheitsgefährdungen und die Clusterabklärung zeitnah und parallel erfolgen.

Bei der Frage nach der Plausibilität eines möglichen Zusammenhangs zwischen Erkrankungsfällen und Exposition (ggf. Literaturrecherche und -bewertung) sind zudem mögliche Expositionspfade und -ereignisse zu erörtern, die mit dem Auftreten der Erkrankungsfälle zeitlich konsistent sind [18]. Bei der Zusammenhangsanalyse kann auch eine Differenzierung in verschiedene Teilperioden oder Teilregionen innerhalb des Untersuchungsgebietes sinnvoll sein.

Internetbasierte Informationssysteme, wie die nachfolgend genannten, liefern Hintergrundwissen zu verschiedenen bekannten Noxen:

- NIS: Noxen-Informationssystem für den öffentlichen Gesundheitsdienst (http://www.nis.nrw.de),

- GDL: Gefahrstoffdatenbank der Länder (http://www.gefahrstoff-info. de/),

— IGS: Informationssystem für gefährliche Stoffe (http://www.stoffliste.de),

- GESTIS: Gefahrstoffinformationssystem der Deutschen Gesetzlichen Unfallversicherung (http://www.dguv. de/bgia/stoffdatenbank),

- ESIS: Europäisches Chemikalien Informations-System (European chemical Substance Information System; http://ecb.jrc.it/esis, Bereich: ORATS),

- TOXNET: Internationale Datenbank zu Toxikologie, Gefährlichen Chemikalien, Umweltmedizin und Giftunfällen (Databases on toxicology, hazardous chemicals, environmental health, and toxic releases; http:// toxnet.nlm.nih.gov, Bereich ITER), 
- ATSDR: Agency for Toxic Substances and Disease Register der USA, angebunden an das National Institute for Health mit einer ausführlichen Übersicht zur Toxikologie und zur möglichen Bevölkerungsexposition,

- U.S. EPA: Environmental Protection Agency der USA mit z. T. sehr ausführlichen Stoffbeschreibungen und Expositions- und Risikoabschätzungen.

Webbasierte Informationen zu gesundheitsgefährdenden Stoffen können auch abgerufen werden über

- BfR: Bundesinstitut für Risikobewertung, http://www.bfr.bund.de oder

- RAPEX: Europäisches Schnellwarnsystem für gefährliche Produkte, die keine Lebensmittel sind, http://ec. europa.eu/consumers/dyna/rapex/ rapex_archives_de.cfm

Hinweise zur Wahrscheinlichkeit eines möglichen Zusammenhanges zwischen einer Umweltquelle und Krebserkrankung geben die vom IARC (International Agency for Research on Cancer) zusammengestellten Monographien: http://monographs.iarc.fr/ und die stoffbezogenen Dokumentationen des ATSDR (Agency for Toxic Substances and Disease Registry, CDC/Atlanta USA): http://www.atsdr.cdc. gov/

\section{Clusteruntersuchungen im engeren Sinne}

Eine detaillierte Clusteruntersuchung umfasst zum einen die Fallvalidierung, zum andern die Ergänzung der Informationen und die nähere Beschreibung des Clusters. Im Gegensatz zur vorläufigen Evaluation wird dabei auch die mögliche Exposition durch Umweltfaktoren näher untersucht. Es sind mithin drei Bereiche zu unterscheiden:

- Bestätigung der Fallzahlerhöhung über die erwartete Fallzahl hinaus,

- nähere Untersuchung der verdächtigten Risikoquelle und der Expositionsbedingungen in der Bevölkerung,

- Überlegungen zur Plausibilität eines Zusammenhanges.
Der erste Arbeitsschritt umfasst zunächst die Validierung der genannten (vorläufigen) Fälle. Dabei sind - mit entsprechenden Einwilligungserklärungen - die den Fall betreffenden ärztlichen Unterlagen zu überprüfen oder aber geeignete Registerdaten abzugleichen. Neben der Validierung der bislang bekannten Fälle ist nach möglichen weiteren Fällen zu forschen, um die Fallliste zu vervollständigen. Die in der vorläufigen Evaluation verwendeten Referenzzahlen sowie Nennerangaben sind ggf. ebenfalls zu vervollständigen. Den Abschluss bildet die Reanalyse der in der vorläufigen Evaluation durchgeführten Statistiken. Sofern die Fallzahl, die Fragestellung und die Datenlage es zulassen, können bereits auch spezifische Verfahren der räumlichen Statistik, ggf. auch Prüfungen der Homogenität des Risikos auf der Ebene der Wohnortkoordinaten eingesetzt werden [31].

Das spezifische Vorgehen ist dabei stark von den vorliegenden Informationen und der Art des Clusterverdachtes abhängig, sodass die folgenden Schritte nur umrissen werden können. Auf jeden Fall ist auf dieser Stufe der Clusterabklärung die Kooperation zwischen verschiedenen Fachdisziplinen zwingend notwendig. Für die Organisation des Abklärungsprozesses muss eine kompetente Stelle mit entsprechender Infrastruktur ausgewählt werden, bei der alle Informationen zusammenfließen, die gleichzeitig die Zusammenführung der Kompetenz aus verschiedenen Fachdisziplinen und Fachbereichen organisiert und eine angemessene und zeitnahe Kommunikation nach außen gewährleistet. Hierfür sind Infrastruktur und Finanzmittel erforderlich.

Wird für die gemeldete Krankheitshäufung ein epidemiologischer Anfangsverdacht bestätigt und ist eine mögliche Umweltquelle, wie z. B. eine Sondermülldeponie oder industrielle Anlage benannt, sollten, sofern möglich, auch Proben bzw. Messungen zur Charakterisierung des Ausmaßes einer Umweltbelastung in die Untersuchung einbezogen werden. In Abhängigkeit von der/den krankheitsspezifisch relevanten Noxe/ $\mathrm{n}$ bringen Beprobungen von Wasser, Boden und Luft zur Bestimmung der Belastung dieser Umweltmedien ggf. weitere Hinweise zur Charakterisierung der Umweltquelle.
Hierzu müssen regionale und überregionale Untersuchungsämter, wie z. B. Landesämter für Umwelt, in die Aufklärungsbemühungen einbezogen werden. Wird von einer im Körper kumulierenden Substanz oder Substanzgruppe (mit langer biologischer Eliminationshalbwertzeit) als möglichem Verursacher ausgegangen, so sollte auch der Einsatz von Methoden des Humanbiomonitorings [32] erwogen werden.

Die Untersuchung der verdächtigen Exposition kann sowohl aktuelle Bodenoder Wasserbeprobungen umfassen als auch die Durchsicht von Emissions- oder Immissionswerten der zurückliegenden Jahre. Einen detaillierten Rahmen für Ablaufpläne von entsprechenden Clusterverdachtsuntersuchungen gibt $z$. B. die Arbeit des neuseeländischen Gesundheitsministeriums [19]. Ziel muss sein, nicht nur eine aktuelle Expositionsabschätzung zu ermöglichen, sondern überdies auch Aussagen über ein zurückliegendes Expositionsniveau und dessen zeitliche Stabilität bzw. Änderung. Dies ist aufgrund der anzunehmenden Latenzzeit zwischen einer relevanten Exposition und dem Ausbruch bzw. der Diagnose der betrachteten Erkrankungen wesentlich. Neben Messungen und der Auswertung bestehender Daten können somit auch Extrapolationsoder Ausbreitungsrechnungen die expositionsseitigen Clusteruntersuchungen vervollständigen.

In der Phase der Validierung des Clusters und der beginnenden Überprüfung möglicher externer Ursachen müssen auch mögliche medizinische Risikofaktoren berücksichtigt werden. Vorerkrankungen sowie frühere medikamentöse oder strahlentherapeutische Behandlungen, die nach dem Stand der Wissenschaft mit der untersuchten Krankheit assoziiert sind, sollten nach Möglichkeit von jedem der Betroffenen innerhalb des Krankheitsclusters erhoben werden. Eine Familienanamnese bei den Betroffenen erweitert das Spektrum möglicher anderer Einflussfaktoren (familiäre Häufung bestimmter Krankheiten etc.), die in die Validierung einbezogen werden können.

An dieser Stelle muss überlegt werden, ob zur Aufklärung des Clusters die Durchführung einer analytischen epidemiologischen Studie $[20,31]$ notwendig ist. 
In Situationen, in denen allein wegen zu geringer Fallzahlen die Durchführung einer Studie nicht möglich ist, aber gleichzeitig aus den gesammelten Daten ein medizinisch begründbarer oder aus der fachlichen Erfahrung plausibler Hinweis auf eine Verursachung durch äußere Einflüsse vorliegt, muss der Clusterverdachtsmeldung gleichwohl angemessen nachgegangen werden. Dies sollte durch die Darstellung der Fälle im Rahmen einer Kasuistik erfolgen, die in die etablierten Meldewege gegeben wird. Liegen gefahrenrechtlich relevante Hinweise vor, so sind die entsprechenden staatlichen Organe ebenso wie die eigene Rechtsabteilung unmittelbar in das Verfahren einzubeziehen.

Durch die direkte Ansprache und Nachfragen bei der regionalen Ärzteschaft bzw. ihren Kammern und Verbänden können kleinräumig aufgefallene Häufungen im Hinblick auf ihre regionale Verbreitung orientierend geprüft werden. Bei einer angenommenen Verursachung durch Chemikalien, Haushaltsprodukte oder Kosmetika ebenso wie bei einer vermuteten Umweltkontamination sollte dies als Meldung entsprechend $₫ 16$ ChemG [33] an das Bundesinstitut für Risikobewertung (BfR) erfolgen. Hierbei sollten ggf. auch die Vergiftungszentralen der Länder informiert werden (Telefon- und Adressliste z. B.: http:// www.bfr.bund.de/cm/252/verzeichnis_ der_giftinformationszentren.pdf).

\section{Exkurs: systematische Dokumentation von Kasuistiken}

Im Fall einer vermuteten infektiösen Übertragung oder einer Infektionskrankheit durch Erreger aus tierischen Reservoiren, die durch Lebensmittel auf Menschen übertragen werden können, sind die Meldewege und Verfahren entsprechend IfSG anzuwenden [34]. Ohne eine systematische Erfassung der Fallzahlen ist auch hier eine Beurteilung von zeitlichen Trends und von regionalen Entwicklungen kaum möglich.

Die Krankheits- und Krebsregister der Länder, ggf. auch das Deutsche Kinderkrebsregister (http://www.kinderkrebsregister.de) und die Landesgesundheitsämter, sollten durch die Weiterleitung der Kasuistiken informiert werden, wenn eine angenommene Häufung von Fällen mit einem Ursachenverdacht vorliegt. Da in übergeordneten Institutionen der Länder und des Bundes Informationen aus unterschiedlichen Regionen und Zeitperioden zusammenlaufen, können unerwünschte zeitliche Entwicklungen, die jeweils räumlich getrennt mit niedrigen Fallzahlen auftreten, dort eher und schneller erkannt werden. Fallbezogene, gut dokumentierte Kasuistiken haben hier einen eigenständig hohen wissenschaftlichen Stellenwert in der Aufklärung von Krankheitsursachen. Nach Informationsweitergabe ist die Chance deutlich größer, dass die lokal nicht lösbare Problematik überregional eingeordnet wird und in epidemiologischen Studien mit einer größeren Fallzahl aufgegriffen wird.

\section{Abgeleiteter Forschungsbedarf aus einer Clusteruntersuchung}

Sofern sich in der Clusteruntersuchung die Fallhäufung bestätigt und eine bislang als Risikofaktor nicht bestätigte Exposition nach wie vor als für die Häufung plausibel erscheint, sollte entschieden werden, ob sich ein konkreter Forschungsbedarf ergibt. Dabei wäre in einer Machbarkeitsstudie zu klären, ob sich zusammen mit anderen Regionen, die vergleichbare Expositionsbedingungen aufweisen, eine gemeinsame Untersuchung mit ausreichenden Erfolgsaussichten („Power“) durchführen lässt. Beispielsweise gibt das Washington State Department [22] für diesen Übergang zur ätiologischen Forschung die folgenden Kriterien vor:

Fallzahl: mindestens fünf gleichartige Fälle.

Risikoerhöhung: Das Verhältnis von „beobachteten“ $[\mathrm{O}] \mathrm{zu}$ „erwarteten“ Fällen [E] soll bei 50 oder mehr beobachteten Fällen mindestens 2 betragen. Bei weniger Fällen soll nach der dort genutzten Berechnungsformel das Verhältnis von „,beobachteten“ [O] zu „erwarteten“ Fällen mindestens 100 dividiert durch die beobachtete Fallzahl betragen $[\mathrm{O} / \mathrm{E}$ $\geq(100 / \mathrm{O})]$.

Public Health Relevanz: Die Untersuchung ist für die öffentliche Gesundheitsvorsorge relevant. Weitergehende Untersuchungen wären unnötig, falls die potenziell relevanten Expositionen beseitigt sind.

Plausibilität der Exposition: Bei bekannter oder verdächtigter Ätiologie: Es muss eine Belastung, die die Erhöhung plausibel verursacht haben könnte, vorhanden sein. Bei unbekannter Ätiologie: Es liegt eine einzigartige Exposition mit plausiblem Expositionspfad vor und nach der wissenschaftlichen Literatur gilt eine Assoziation zwischen Exposition und Erkrankung nicht als unwahrscheinlich.

Nach diesen Kriterien würde die regionale Clusteruntersuchung $[35,36,37]$ in eine vorab geplante epidemiologische Studie mit Studiendesign und Auswerteplan überführt (3. Phase der Clusterabklärung, siehe $-A b b .2)$. Eine Beendigung der regionalen Clusteruntersuchung ergibt sich zum einen daraus, dass die Kriterien für den Übergang zu einer intensiveren Bearbeitungsstufe nicht erfüllt sind oder dass zum anderen im Verlauf der Untersuchung ein bereits bekannter Risikofaktor identifiziert und im Sinne einer Risiko-/ Gefahrenabwehr beseitigt werden konnte. Einen Überblick über die möglichen Entscheidungen bei einer Clusteruntersuchung gibt $\bullet$ Abb. 2.

In einigen Fällen kann auf eine Clusteruntersuchung aber auch verzichtet werden, wenn eine verdächtigte und leicht zu beseitigende Exposition ohne abschlieBenden wissenschaftlichen Nachweis oder ohne wissenschaftlich im Einzelfall belegten Hinweis eines verursachenden Erkrankungsrisikos mit dem Argument abgestellt wird, dass die Kosten einer weitergehenden Untersuchung die Kosten der Beseitigung einer möglicherweise wirksamen Expositionsquelle übertreffen können.

\section{Einschränkungen des Aussagegehaltes von Clusteruntersuchungen}

Wenn in einer Region, einer Gemeinde oder einem Ortsteil eine erhöhte Erkrankungs- oder Mortalitätsrate beobachtet oder vermutet wird und wenn von der betroffenen Bevölkerung eine nähere Untersuchung dieser Häufung gefordert 
wird, stellt sich in der öffentlichen und wissenschaftlichen Debatte immer wieder die Frage nach Möglichkeiten und Einschränkungen kleinräumiger Untersuchungen $[38,39]$. Rothman [40] hat die wissenschaftlichen Vorbehalte gegen Clusterverdachtsuntersuchungen in folgenden Punkten zusammengefasst: (i) Einzelne Cluster sind im Allgemeinen zu klein für epidemiologische Untersuchungen. (ii) Den Beobachtungen liegen selten klare Falldefinitionen zugrunde (heterogenes Diagnosenspektrum). (iii) Die Festlegung einer sinnvollen Bezugspopulation ist schwierig und erfolgt oft willkürlich. (iv) Die angeschuldigte(n) Exposition(en) liegen sehr oft im Bereich niedriger Konzentrationen, sind heterogen verteilt und unscharf definiert. (v) Durch das Cluster wird öffentliche Aufmerksamkeit hervorgerufen, die eine unverfälschte Datenerhebung (insbesondere per Fragebogen) erschwert oder unmöglich macht.

In vielen Untersuchungen von Clusterverdachtsmeldungen stellt sich auf Stufe 1 oder Stufe 2 der Prüfung heraus, dass es sich um eine im statistischen Sinn auffällige regionale Erkrankungs- bzw. Mortalitätsrate handelt [21, 24, 41]. In solchen Fällen sind die zuständigen Fachleute oftmals in der schwierigen Situation, erklären zu müssen, dass eine beobachtete Häufung nicht notwendigerweise auf eine gemeinsame Ursache zurückgeführt werden kann und dass auch im Rahmen der zu erwartenden zeitlichen und räumlichen Variation derartige Häufungen auftreten können [42]. In der Öffentlichkeit lösen derartige Erklärungen nicht selten Unverständnis bzw. Zweifel an der Richtigkeit der Aussagen aus. Dies kann sich in entsprechend kritischen Presseberichten oder kontroversen öffentlichen Debatten widerspiegeln oder auch durch solche verstärkt werden. Häufig werden als Reaktion auf solche Aussagen durch Bürgerinitiativen weitere Untersuchungen gefordert oder eigene Erhebungen angestrebt.

Eine mögliche Verzerrung von epidemiologischen Untersuchungen durch hohe öffentliche Aufmerksamkeit stellt ein ernsthaftes Problem für vertiefte Clusteruntersuchungen dar, weil die Erhebung von Symptomen oder Erkrankungen in betroffenen Gebieten häufig vollständiger als in nicht betroffenen (Vergleichs-)Regionen ausfällt und/oder eine kontroverse, politisch polarisierte öffentliche Diskussion zu einer Teilnahmeverweigerung einzelner Gruppen führen kann. Ein weiteres Problem stellen ggf. kleine Fallzahlen dar. Rothman [40] kommt zu dem Schluss, dass aufgrund der aufgeführten Vorbehalte der wissenschaftliche Wert von Clusteruntersuchungen zur Aufklärung der Ätiologie von Erkrankungen als nicht sehr hoch einzuschätzen ist. Da es jedoch zu der hier beschriebenen Vorgehensweise keine etablierte und begründbare Alternative gibt und die Bedeutung von zeitlichregionalen Häufungen nie vorab einzuschätzen ist, muss die sachgerechte Bearbeitung entsprechender Anlässe in die Routinearbeiten auf lokaler Ebene aufgenommen werden.

Auch wenn sich in der gestuften Prüfung des Clusterverdachts im statistischen Sinn eine Auffälligkeit zeigt, bedeutet dies nicht, dass die Häufung von Erkrankungen, das Cluster, fachlich erklärbar sein muss. Während bei infektionsepidemiologisch zu erklärenden Krankheitshäufungen bei gesichertem Fachwissen auch bei (noch) nicht aufgeklärten Übertragungswegen die wahrscheinlichen Übertragungsmechanismen benannt und vielfach auch Hinweise zu präventivem Verhalten und Handeln gegeben werden können, sind in umweltmedizinischen Cluster-Fragestellungen oft weder die Einflussgrößen (die Noxen, die Expositionsstärke und -dauer) noch die Wirkungsweise (die Dosis-WirkungsBeziehung, die altersabhängige Empfindlichkeit, die Latenzzeit) wissenschaftlich gesichert. Ausgehend von einer Feststellung, dass eine wenig wahrscheinliche zeitlich-räumliche Häufung von Erkrankungen vorliegt, wird nach zeitlich vorausgehenden Ursachen in der Lebensumwelt gesucht. Der 1791 formulierte Kantsche Lehrsatz [43] „Wenn wir erfahren, dass etwas geschieht, so setzen wir dabei jederzeit voraus, dass etwas vorhergehe, worauf es nach einer Regel folgt" fordert zur wissenschaftlichen Erklärung von Auffälligkeiten auf. Kant enttarnt dabei das „Zufällige als erklärendes Moment“ als „Unwissen über die Zusammenhänge (die Regeln)“ bzw. als
„Unkenntnis der vorausgegangenen Bedingungen“. In Clusterbetrachtungen muss vielfach beides eingestanden werden.

\section{Zusammenfassung}

Der Umgang mit und das Management von Untersuchungen zur Abklärung zeitlich-räumlicher Erkrankungshäufungen (Cluster) fordert eine „Balance zwischen Wissenschaft und Öffentlichkeitsarbeit" [41]. Jede neu gemeldete Häufung von Fällen kann auf ein ernstes Problem hindeuten, jede Meldung muss daher sachgerecht, zeitnah und mit anerkannten wissenschaftlichen Methoden bearbeitet werden. Gleichzeitig zeigt die Erfahrung vieler Institutionen, dass die überwiegende Zahl von Meldungen sich nicht in einen begründeten Clusterverdachtsfall, dem vertieft nachzugehen wäre, verwandelt. $\mathrm{Da}$ der erforderliche Klärungsprozess mit einem hohen Zeit-, Infrastruktur- und Personalaufwand ebenso wie mit hohen Anforderungen an die epidemiologische Kompetenz verbunden ist, besteht die Gefahr, dass auch berechtigte Anfragen und Hinweise ggf. nicht angemessen aufgenommen und bearbeitet werden, die erforderliche Kooperation zwischen unterschiedlichen Diensten und Institutionen nicht gesucht wird und die Kommunikation zwischen Bürgern, der niedergelassenen Ärzteschaft und den kommunalen Gesundheitsverwaltungen nicht gepflegt wird. Da sich jederzeit alte und bestehende Risikoquellen in zeitlich-räumliche Häufungen von adversen Effekten verwandeln können und da das Auftreten neuer Quellen nicht auszuschließen ist, muss dieser Entwicklung entgegengetreten werden.

Die RKI-Kommission „Methoden und die Qualitätssicherung in der Umweltmedizin“ möchte mit dieser Empfehlung dazu beitragen, dass

- gesehene oder vermutete Cluster sachgerecht, effektiv und zielführend bearbeitet werden,

- der Kenntnisstand über bestehende Infrastruktur und Informationsquellen verbessert wird,

- die beteiligten Institutionen ermutigt werden, sich szenarisch und organisatorisch auf entsprechende Probleme vorzubereiten, 
- zeitnah die notwendigen Daten und Informationen zwischen den Institutionen ausgetauscht werden,

- Untersuchungen durch den Erhalt bzw. Aufbau notwendiger personeller und finanzieller Ressourcen auf Ebene der zuständigen Kommunalund Landesbehörden effektiv durchgeführt werden können,

- das schrittweise Vorgehen vereinheitlicht und damit für alle Beteiligten transparenter und nachvollziehbarer wird und

- durch eine gute Dokumentation und Risikokommunikation das Vertrauen der Öffentlichkeit in die Vorgehensweise bei beobachteten oder vermuteten zeitlich-räumlichen Häufungen von Erkrankungen erhöht wird.

Die Länder werden gebeten, die Kommissionsempfehlung an ihre länderspezifischen Strukturen und Besonderheiten anzupassen. Zur weiteren Vertiefung der Thematik und der methodischen Verfahren wird auf die angefügte Literatur verwiesen.

\section{Autorengruppe}

Dr. Michael Schümann (Federführung und Koordination), Behörde für Soziales, Gesundheit und Verbraucherschutz Hamburg, Umwelt und Gesundheit, Billstraße 80a, 20539 Hamburg

PD Dr. Gabriele Bolte, MPH, Bayerisches Landesamt für Gesundheit und Lebensmittelsicherheit, Sachgebiet Umweltmedizin, Veterinärstr. 2, 85764 Oberschleißheim

Dr. Birger Heinzow, Landesamt für soziale Dienste, Dezernat 34, Umweltbezogener Gesundheitsschutz, Brunswikerstr. 4, 24105 Kiel

Dipl.-Stat. Michael Hoopmann, Niedersächsisches Landesgesundheitsamt, Abt. Umweltmedizin/Epidemiologie, Roesebeckstr. 4-6, 30449 Hannover

Dr. Peter Kaatsch, Universitätsklinikum Mainz - Deutsches Kinderkrebsregister, Institut für Medizinische Biometrie, Epidemiologie und Informatik (IMBEI), Obere Zahlbacher Straße 69, 55131 Mainz

Dr. Roland Suchenwirth, Niedersächsisches Landesgesundheitsamt, Abt. Um-
weltmedizin/Epidemiologie, Roesebeckstr. 4-6, 30449 Hannover

Dr. Claudia Terschüren, MPH, Landesinstitut für Gesundheit und Arbeit NRW, Zentrum für Öffentliche Gesundheit, Westerfeldstr. 35-37, 33611 Bielefeld

Dr. Iris Zöllner, Epidemiologie und GBE, Regierungspräsidium Stuttgart, Landesgesundheitsamt, Nordbahnhofstr. 135, 70191 Stuttgart

\section{Externe Sachverständige}

Dr. Stefan Hentschel, Hamburgisches Krebsregister, Billstraße 80a, 20539 Hamburg

PD Dr. Alexander Katalinic, Institut für Krebsepidemiologie, Krebsregister Schleswig-Holstein, Universität zu Lübeck, Beckergrube 43-47, 23552 Lübeck

Dr. Klaus Kraywinkel, Epidemiologisches Krebsregister NRW, Robert-KochStraße 4O, 48149 Münster

\section{Kommissionsmitglieder}

Dr. A. Beyer (Umweltmed. Ambulanz Berlin-Steglitz/Zehlendorf), Prof. Dr. W. Dott (Universitätsklinikum Aachen, Institut für Hygiene und Umweltmedizin), Prof. Dr. H. Drexler (Friedrich-Alexander-Universität Erlangen-Nürnberg, Institut für Arbeits-, Sozial- und Umweltmedizin), Prof. Dr. H. Dunkelberg (Universität Göttingen, Abt. Allg. Hygiene u. Umweltmedizin), Prof. Dr. Th. Eikmann (Universität Gießen, Institut f. Hygiene u. Umweltmedizin), Dr. B. Heinzow (Landesamt für soziale Dienste Schleswig-Holstein, Dezernat Umweltbezogener Gesundheitsschutz, Kiel), Prof. Dr. C. Hornberg (Universität Bielefeld, Fakultät für Gesundheitswissenschaften), Prof. Dr. Dr. A.D. Kappos (Frankfurt/Main), Prof. Dr. K.E. von Mühlendahl (Kinderhospital Osnabrück, Gemeinnützige Kinderumwelt $\mathrm{GmbH}$ ), Prof. Dr. D. Nowak (LMU München, Klinikum Innenstadt, Institut u. Poliklinik für Arbeits- und Umweltmedizin), PD Dr. F.-A. Pitten (Institut für Krankenhaushygiene und Infektionskontrolle GbR, Gießen), Dr. W. Stück (Ökologischer Ärztebund/ISDE, Koblenz), Prof. Dr. M. Schwenk (Tübingen), Dr. R. Suchenwirth (Niedersächsisches Landesgesundheitsamt, Abt. Umweltmedizin/Epidemiologie, Hannover), Prof. Dr. M. Wil- helm (Universität Bochum, Hygiene, Sozial- und Umweltmedizin).

\section{Ständige Gäste}

Dr. N. Englert (Umweltbundesamt, Berlin), Dr. A. Hahn (Bundesinstitut für Risikobewertung, Berlin), Dr. U. Winkler (Bundesministerium für Gesundheit, Referat 332).

\section{Geschäftsstelle im RKI}

Dr. D. Eis, Dr. U. Wolf.

\section{Literatur}

1. WHO/IPCS - International Program for Chemical Safety (1994) Assessing human health risks of chemicals: derivation of guidance values for health-based exposure limits. Environmental Health Criteria (EHC) 170, World Health Organization, Geneva

2. HmbGDG (2001) Gesetz über den Öffentlichen Gesundheitsdienst in Hamburg (Hamburgisches Gesundheitsdienstgesetz - HmbGDG) vom 18. Juli 2001 (hier insb. § 15). HmbGVBI. 2001, S. 201 (letzte Änderung 14. Dez. 2007 in HmbGVBI. 2008, S. 17)

3. NRC (1989) Improving risk communication. Committee on Risk Perception and Communication and Commission on Physical Sciences, Mathematics, and Applications, National Research Council. Washington, DC, National Academy Press, http://www.nap.edu/books/0309039436/html/

4. Fiore BJ, Hanrahan LP, et al. (1990) State health department response to disease cluster reports: a protocol for investigation. Am J Epidemiol 132(1 Suppl):S14-S22

5. Trumbo CW (2000) Public requests for cancer cluster investigations: a survey of state health departments. Am J Public Health 90:1300-1302

6. CDC (1990) Guidelines for investigating clusters of health events. MMWR Recommendations and Reports 39 (RR-11): 1-16, http://www.cdc.gov/ $\mathrm{mmwr} /$ preview/mmwrhtml/00001797.htm

7. GEP (2008) Leitlinien und Empfehlungen zur Sicherung von Guter Epidemiologischer Praxis (GEP)/Langversion. Deutsche Gesellschaft für Epidemiologie (DGEpi) in Zusammenarbeit mit der Deutschen Gesellschaft für Medizinische Informatik, Biometrie und Epidemiologie (GMDS), Deutschen Gesellschaft für Sozialmedizin und Prävention (DGSMP), Deutschen Region der Internationalen Biometrischen Gesellschaft (DRIBS), Fassung: Juli 2008. http://www.dgepi.de/ infoboard/stellungnahmen.htm

8. Brès $P$ (1986) Public health action in emergencies caused by epidemics. A practical guide. WHO World Health Organization, Geneva

9. Snow J (1857) Über die Verbreitungsweise der Cholera. Deutsche Übersetzung der 2. Ausgabe "On the Mode of Communication of Cholera", London 1854. Verlag H.C. Huch, Quedlinburg

10. CDC (1982) A cluster of Kaposi's sarcoma and pneumocystis carinii pneumonia among homosexual male residents of Los Angeles and Orange Counties, California. MMWR Morb Mortal Wkly Rep 31(23): 305-307, http://www.cdc.gov/mmwr/ preview/mmwrhtml/00001114.htm 
11. Greaves MF (1997) Aetiology of acute leukaemia. Lancet 349:344-349

12. O'Connor SM, Boneva RS (2007) Infectious etiologies of childhood leukemia: plausibility and challenges to proof. Environ Health Perspect 115:146-150

13. Ministry of the Environment, Government of Japan (2002) Minamata disease: the history and measures. Environment and Health Department. Tokyo, Japan, http://www.env.go.jp/en/chemi/hs/ minamata2002/ch3.html

14. NCl (2006) Cancer clusters. Fact sheet. National Cancer Institute, U.S. National Institutes of Health. http://www.cancer.gov/cancertopics/factsheet/ Risk/clusters

15. Newhouse M, Thompson H (1965) Mesothelioma of pleura and peritoneum following exposure to asbestos in the London area. Br J Ind Med 22: 261-269

16. Fleming LE, Ducatman AM, Shalat SL (1992) Disease clusters in occupational medicine: a protocol for their investigation in the workplace. Am J Ind Med 22:33-47

17. Wildner M, Heißenhuber A, Bolte G (2004) Cluster - Die epidemiologische Bewertung räumlicher und zeitlicher Häufungen. Fachinformation Gesundheit. Bayerisches Landesamt für Gesundheit und Lebensmittelsicherheit, Erlangen, http:// www.Igl.bayern.de/publikationen/index.htm

18. MDCH (2007) Chemical illness response: guidelines for public health investigations of acute onset illness clusters of chemical etiology. Michigan Department of Community Health, U.S.A. September 28

19. Ministry of Health (1997) Investigating clusters of non-communicable disease. Guidelines for public health services, Ministry of Health, Wellington, New Zealand, http://www.moh.govt.nz/moh.nsf/ Files/cluster/\$file/cluster.pdf

20. Rothenberg RB, Thacker SB (1992) Guidelines for the investigation of clusters of adverse health events. In: Elliott P, Cuzick J, English D, Stern R (eds) Geographical and environmental epidemiology: methods for small-area studies. Oxford University Press, Oxford

21. Thun MJ, Sinks T (2004) Understanding cancer clusters. CA Cancer J Clin 54:273-280

22. Washington State Department of Health (2007) Guidelines for investigating clusters of chronic disease and adverse birth outcomes. Revised July 2007. Washington, DC, Washington State Department of Health. http://www.doh.wa.gov/EHSPHL/ Epidemiology/NICE/publications/ClusterProtocol. pdf

23. Wildner M, Fischer R, Bolte G (2006) Cluster: Grundlagen der epidemiologischen Bewertung räumlicher und zeitlicher Häufungen - das Fallbeispiel Diabetes-Cluster. Umwelmed Forsch Prax 11:223-230

24. Kingsley BS, Schmeichel KL, Rubin CH (2007) An update on cancer cluster activities at the Centers for Disease Control and Prevention. Environ Health Perspect 115(1):165-171

25. Gesetz zur Verhütung und Bekämpfung von Infektionskrankheiten beim Menschen (Infektionsschutzgesetz, IfSG). Infektionsschutzgesetz vom 20. Juli 2000 (BGBI. I S. 1045), zuletzt geändert durch Artikel 2 des Gesetzes vom 13. Dezember 2007 (BGBI. I S. 2904)

26. Bolte G, Büchele G, Schwegler U, et al. (2006) Kleinräumige Prävalenzunterschiede von Atemwegserkrankungen bei Kindern: Grenzen der Erkenntnismöglichkeiten einer von Eltern angeregten Studie. Gesundheitswesen 68:760-768
27. Kaatsch $P$ (2006) Empfehlungen für eine gute Risikokommunikation - Erfahrungen anhand deutscher Studien zu Krebs bei Kindern und Wohnortnähe zu Kernkraftwerken. Umweltmed Forsch Prax 11:27-31

28. Quataert P, Armstrong B, Berghold A, et al. (1999) Methodological problems and the role of statistics in cluster response studies: a framework. Eur J Epidemiol 15:821-831

29. Drijver M, Woudenberg F (1999) Cluster management and the role of concerned communities and the media. Eur J Epidemiol 15:863-869

30. Neutra R (1990) Counterpoint from a clusterbuster. Am J Epidemiol 132:1-8

31. Elliott P, Savitz DA (2008) Design issues in smallarea studies of environment and health. Environ Health Perspect 116:1098-1104 (doi:10.1289/ ehp.10817; available at http://dx.doi.org/)

32. UBA (2007) Humanbiomonitoring. Zugang zu empfohlenen Verfahren und Referenzwerten unter: http://www.umweltbundesamt.at/ umweltschutz/gesundheit/ges_aktionen/ humanbiomonitoring/

33. Gesetz zum Schutz vor gefährlichen Stoffen (Chemikaliengesetz, ChemG): Chemikaliengesetz in der Fassung der Bekanntmachung vom 2. Juli 2008 (BGBI. I S.1146)

34. BfR (2007) Untersuchung lebensmittelbedingter Ausbrüche - Handbuch zu den Erfassungsbögen für Lebensmittel. Bundesinstitut für Risikobewertung, Berlin

35. Hoffmann W, Dieckmann H, Dieckmann H, Schmitz-Feuerhake I (1997) A cluster of childhood leukemia near a nuclear reactor in northern Germany. Arch Environ Health 52(4):275-280

36. Hoffmann W, Terschueren C, Richardson DB (2007) Childhood leukemia in the vicinity of the Geesthacht nuclear establishments near Hamburg, Germany. Environ Health Perspect 115(6):947-952

37. Hoffmann W, Terschüren C, Heimpel H, et al. (2008) Population-based research on occupational and environmental factors for leukemia and nonHodgkin's lymphoma: the Northern Germany Leukemia and Lymphoma Study (NLL). Am J Ind Med 51(4):246-257

38. Wartenberg D (1995) Should we boost or bust cluster investigations? Epidemiology 6:575-576

39. Wartenberg D (2002) Investigating disease clusters: why, when and how? J R Statistical Soc: Series A (Statistics in Society) 164(1):13-22

40. Rothman K (1990) A sobering start to the clusterbusters conference. Am J Epidemiol 132:6-12

41. Oregon State Cancer Registry - OSCaR (2007) Cancer clusters: common or chance. http://www. oregon.gov/DHS/ph/oscar/clustercommonchance. shtml

42. Zöllner I (1997) Probleme und Möglichkeiten kleinräumiger Untersuchungen zu seltenen Erkrankungen. In: Manikowsky S von, BaumgardtElms C, Schümann M, Haartje U (Hrsg) Methoden regionalisierter Beschreibung und Analyse von Krebsregisterdaten. Ed. Temmen, Bremen

43. Kant I (1791/1998) Kritik der reinen Vernunft/ Analytik der Grundsätze/2. Hauptstück 171. Felix Meiner, Hamburg 